\title{
Distractor Interference During Smooth Pursuit Eye Movements
}

\author{
Miriam Spering and Karl R. Gegenfurtner \\ Justus-Liebig-Universität Giessen
}

\author{
Dirk Kerzel \\ Université de Genève
}

\begin{abstract}
When 2 targets for pursuit eye movements move in different directions, the eye velocity follows the vector average (S. G. Lisberger \& V. P. Ferrera, 1997). The present study investigates the mechanisms of target selection when observers are instructed to follow a predefined horizontal target and to ignore a moving distractor stimulus. Results show that at $140 \mathrm{~ms}$ after distractor onset, horizontal eye velocity is decreased by about $25 \%$. Vertical eye velocity increases or decreases by $1 \%$ in the direction opposite from the distractor. This deviation varies in size with distractor direction, velocity, and contrast. The effect was present during the initiation and steady-state tracking phase of pursuit but only when the observer had prior information about target motion. Neither vector averaging nor winner-take-all models could predict the response to a moving to-be-ignored distractor during steady-state tracking of a predefined target. The contributions of perceptual mislocalization and spatial attention to the vertical deviation in pursuit are discussed.
\end{abstract}

Keywords: eye movements, smooth pursuit, vector averaging, inhibition, attention

Smooth pursuit eye movements are used to stabilize a moving object of interest on the fovea or to keep the environment stable during linear self-motion. In this article, we investigate the integration of motion information from two identical stimuli moving in different directions, when the observer has prior information about which stimulus to pursue.

\section{Background on Motion Integration for Pursuit Eye Movements: Information Averaging}

The mechanisms involved in selecting a single, moving object from among several alternatives have been examined previously (e.g., Lisberger \& Ferrera, 1997; Recanzone \& Wurtz, 1999, 2000). In these studies, the smooth pursuit system had to choose between two simultaneously presented visual objects that moved into different directions. Results on the effect of the two associated motion vectors on pursuit behavior are largely consistent (see Born, Pack, \& Zhao, 2002; Heinen \& Keller, 2004). During pursuit initiation (open-loop phase), the eye movement response followed a vector average of the velocity of the two objects (Lisberger \&

Miriam Spering and Karl R. Gegenfurtner, Allgemeine Psychologie, Justus-Liebig-Universität Giessen, Giessen, Germany; Dirk Kerzel, Faculté de Psychologie et des Sciences de l'Education, Université de Genève, Genève, Switzerland.

This research was supported by the Deutsche Forschungsgemeinschaft Graduate Program, Brain and Behavior Grant DFG 885/1. Karl R. Gegenfurtner was supported by the Deutsche Forschungsgemeinschaft Forschergruppe, Perception and Action Grant DFG 560. Dirk Kerzel was supported by SNF 10011-107768/1. We thank Stephen Lisberger, Guillaume Masson, John Maunsell, and Werner Schneider for valuable comments and suggestions. We also thank Doris Braun, Nina Barthel, Lars Pracejus, and Martin Stritzke, who tested the experiments and participated in all of them.

Correspondence concerning this article should be addressed to Miriam Spering, Justus-Liebig-Universität Giessen, FB 06 Psychologie, Allgemeine Psychologie, Otto-Behaghel-Strausse 10F, 35394, Giessen, Germany. E-mail: miriam.spering@psychol.uni-giessen.de
Ferrera, 1997). In particular, vector averaging occurred when both objects had identical appearance and the animal or human observer had no prior information about which stimulus to choose. It is interesting to note that vector averaging was also the dominant response during the open-loop phase, when the two objects differed in their physical properties (Lisberger \& Ferrera, 1997) or when the animal was cued to pursue one of the two objects (Ferrera, 2000; Ferrera \& Lisberger, 1997). A vector averaging pursuit response was also obtained in a study in which only one of the motion signals was visually evoked and the other was produced by microstimulating cortical neurons in the middle temporal visual area (MT; Groh, Born, \& Newsome, 1997). When prior information about the identity of the to-be-pursued object (the target) was given, animals and human observers could use selective attention to suppress the response to the irrelevant object (the distractor). The result was a winner-take-all pursuit response (Ferrera \& Lisberger, 1997; Kowler, 1990). However, Recanzone and Wurtz (1999) demonstrated that the attention effect developed only after some time, with a latency of approximately $450 \mathrm{~ms}$. The best prediction of pursuit behavior shifted from a vector average during open-loop pursuit to a winner-take-all model during steady-state pursuit, depending on the spatial and temporal separation of target and distractor in the visual field.

Taken together, these studies imply that velocity information from a target and distractor stimulus is averaged when no prior information about the target identity is given or when prior target information has not yet been used to identify target and distractor. In cases such as these, vector averaging can be considered to be the most efficient, error-minimizing response because it enables observers to respond fast to whichever of the two stimuli will become the target. When a target has already been selected, information from the irrelevant distractor has no influence on the ongoing oculomotor program. Here, a winner-take-all response is clearly more efficient. Note that most studies mentioned so far concentrated on open-loop pursuit, for which up to now there seems to be 
conclusive evidence to support vector averaging under the specified circumstances.

In this study, we focus on the steady-state phase of pursuit, in which visual feedback about the retinal velocity or position of the target is used to control the smooth pursuit response (closed-loop phase). In our experimental paradigm, we present a continuously moving horizontal stimulus (target), and at a random point along its trajectory, a second stimulus (distractor) splits off from the target and moves into a different direction. Although target motion is predefined, onset and direction of the distractor are still unpredictable. One possible strategy that the visual system might use is the integration of target and distractor motion in a vector averaging manner, similar to what has been demonstrated for the open-loop response (e.g., Lisberger \& Ferrera, 1997). However, there are reasons to expect that vector averaging does not prevail in our experiments: The error-minimizing effects of vector averaging do not apply to steady-state pursuit because target selection has already been completed, and visual feedback is used to keep the target stabilized on the fovea. A better strategy would be to ignore or to inhibit the distractor as soon as it appears in order to ensure reliable pursuit of the target.

\section{Alternative Model for Motion Integration: Information Inhibition}

The suppression of irrelevant or to-be-ignored information is not always successful. One of the best documented findings in cognitive psychology is that nontarget information is processed and competes for the control of action, even though observers are instructed to ignore it (see Desimone \& Duncan, 1995). This has been demonstrated by classic interference effects (e.g., Eriksen \& Eriksen, 1974; Stroop, 1935). Interference effects have also been found in reaching and saccadic eye movements to predefined targets in the presence of to-be-ignored distractors (Sheliga, Riggio, \& Rizzolatti, 1994; Tipper, Howard, \& Jackson, 1997). Sheliga et al. (1994) asked observers to attend to a cue to the left or to the right of fixation and to make an upward or downward saccade. The presence of nontarget cues increased the latency of the targetdirected motor response and made the movement trajectory curve away from the attended (lateral) position of the cue. That is, an upward saccade is biased toward the left, if a cue on the right has been attended. With their premotor theory of attention, Rizzolatti, Riggio, Dascola, and Umiltà (1987) claimed that this curvature effect results from an inhibition of response to the nontarget cue. Because the cue had previously been attended, an automatically generated motor response to it had to be suppressed.

Similarly, automatically generated, conflicting motor programs could be inhibited during steady-state pursuit in our experiment. We assume that distractor motion will automatically attract attention to some degree because distractor appearance is a highly salient event, occurring close to the present focus of attention (the pursuit target; Kerzel \& Ziegler, 2005; Khurana \& Kowler, 1987). On the basis of previous studies on pursuit initiation, one could either expect a vector averaging response or a winner-take-all response, indicating that the distractor has no effect or that distractor information was ignored successfully. On the basis of what is known about the open-loop saccadic system and manual responses, one could alternatively expect that distractor information interferes with the ongoing response to the target. A deviation away from the distractor would indicate an inhibition of motor programs associated with the distractor. In the context of smooth pursuit eye movements, inhibition has not been a key concept so far. In this study, we therefore address the question of whether different strategies for motion integration are used during openloop and closed-loop pursuit and whether the response depends on prior information on target motion.

\section{Overview of Present Study}

In a series of 10 experiments, we tested the effect of a moving distractor on horizontal and vertical eye velocity, when target direction was predefined. In Part 1, we examine whether a moving distractor affects eye velocity during the steady-state phase of a pursuit eye movement (Experiment 1), in the same way as during pursuit initiation (Experiments 2-3). We also tested the relevance of prior information about target direction during the initiation phase and either instructed observers as to which stimulus to track (Experiment 2) or did not (Experiment 3). The main findings in response to a distractor were (a) a profound slowing in horizontal eye velocity and (b) a deviation of the vertical velocity component in the direction opposite to the distractor. We obtained this result both during the pursuit open-loop and closed-loop phase but only when the observer had prior information about target direction. In Part 2, we tested whether the effect of a moving distractor on horizontal and vertical eye velocity varied systematically with velocity (Experiment 4), contrast (Experiment 5), and number of distractors (Experiment 6). In Parts 3 and 4, we examine in more detail the possible origins of the distractor interference effect, namely a perceptual error with regard to distractor onset position (Experiment 7), an illusory vertical motion component in the target (Experiment 8), a voluntary shift of covert spatial attention (Experiment 9), and the temporal and spatial predictability of the distractor (Experiment 10).

\section{General Method}

In 10 experiments, we investigated the effect of a moving distractor on horizontal and vertical eye position and velocity. The distractor was presented while human observers tracked a predefined moving target with their eyes. In the following section, we summarize the General Method relevant to all 10 experiments. We then report the experimental paradigm and results for each experiment separately.

\section{Observers}

The first author and observers from a pool of 15 subjects (age: $M=26.7$, $S D=6.3$ ) participated, all of whom had some previous experience of oculomotor experiments. Observers were students or postgraduates from the University of Giessen and participated with informed consent. Twelve observers were naive to the hypotheses tested. The performance of nonnaive observers was not significantly different from the pattern of performance in naive observers. All observers had normal or corrected-to-normal visual acuity. Because of the high sensitivity of the eye cameras, the eye-tracking procedure was compatible with observers wearing eyeglasses $(N=2)$ or soft contact lenses $(N=1)$. The research described in this article was conducted in conformity with the American Psychological Association ethical standards. Data for each experiment were collected in individual sessions lasting approximately $60 \mathrm{~min}$. Observers completed between one and three sessions for each experiment. The number of observers varied 
between experiments and so did the number of trials that each observer completed.

\section{Experimental Setup}

In all 10 experiments, stimuli were dots with a Gaussian luminance profile $\left(S D=0.3^{\circ}\right)$ with maximum luminance 58 candelas $/ \mathrm{m}^{2}\left(\mathrm{~cd} / \mathrm{m}^{2}\right)$. Stimuli were presented on a uniform gray background with mean luminance $32 \mathrm{~cd} / \mathrm{m}^{2}$, resulting in a Weber contrast of approximately $81 \%$. Before the split of the stimulus, one Gaussian dot was presented; after the stimulus split, there were two dots present. At the stimulus split, where the Gaussian distributions of the two dots overlap, the luminance at each pixel was chosen to be the maximum luminance of the two distributions.

Stimuli were presented on a 21-in. CRT monitor (ELO Touchsystems, Fremont, CA) by an ASUS V8170 (Geforce 4MX 440) graphics board with a refresh rate of $100 \mathrm{~Hz}$. The gamma nonlinearity of the monitor was measured with a Laser 2000 Photometer (Model 370; UDT Instruments, Baltimore, MD) and corrected by using a look-up table. The spatial resolution of the monitor was $1280(\mathrm{H}) \times 1024(\mathrm{~V})$ pixels, and the screen subtended $37 \mathrm{~cm}\left(45^{\circ}\right)$ horizontally and $29.6 \mathrm{~cm}\left(39^{\circ}\right)$ vertically. At a viewing distance of $47 \mathrm{~cm}$, this results in 28 pixels per degree. All experiments were conducted in a dimly lit room.

\section{Eye Movement Recordings and Analysis}

Eye position signals were recorded with a head-mounted, video-based eye tracker (EyeLink II, SR Research Ltd., Osgoode, Ontario, Canada) and were sampled at $250 \mathrm{~Hz}$. To achieve a more stable tracking of eye position, we used a combined corneal reflection and pupil tracking in every observer. The apparatus was calibrated at the beginning of the experiment and recalibrated after each block of trials ( 96 trials at maximum) by instructing the observer to fixate single dots that appeared successively at nine different positions on the monitor. Calibration eye position was validated by repeating the procedure. If the average eye position measured during validation was above $0.35^{\circ}$ deviation from eye position during calibration, the procedure was repeated. Typically, eye position accuracy was on the order of 0.20 to $0.30^{\circ}$ deviation. On the basis of the calibration results, the system automatically chose the best eye, from which we then recorded. At the beginning of each trial, the EyeLink II system performed a drift correction to correct for shifts of the head-mounted tracking system. Observers were seated with their heads stabilized with a chin rest and viewed the display binocularly. Stimulus display and data collection were controlled by a PC.

Eye position traces for individual trials were stored on disk for off-line analysis. Eye velocity signals were obtained by digital differentiation of eye position signals over time. Velocity traces were then smoothed by a Butterworth filter with a $60-\mathrm{Hz}$ cut-off. Saccades in each trace were detected by using a combined position criterion $\left(0.3^{\circ}\right)$ and fixedacceleration cut-off $\left(8750 \% \mathrm{~s}^{2}\right)$. A period of three samples $(12 \mathrm{~ms})$ before and after saccade onset and offset were also excluded. That the algorithm removed all saccades was confirmed by visual inspection of each position and velocity trace along with the stimulus time course. Traces were aligned to the split of the stimulus, and eye position and velocity were averaged in $40-\mathrm{ms}$ bins and analyzed at an interval of $320 \mathrm{~ms}$ after the stimulus split or until the first saccade occurred. We excluded traces from further analysis that were contaminated by eye blinks. Overall, less than $2 \%$ of trials in any of the experiments were excluded (see Experiment 2 for an exception).

For each experiment, eye velocity data in the relevant interval were analyzed across observers. We conducted further statistical tests on the average eye velocity in the time interval after the split (120-160 ms), in which the effect was at its maximum, by using the statistical software package SPSS 11.0 for Windows (SPSS, Inc., 2001).

\section{Part 1: The Basic Findings}

\section{Experiment 1: Effects of a Moving Distractor on Pursuing a Predefined Horizontal Target}

Method. In the first experiment, we explored the effect of a moving distractor on horizontal and vertical eye velocity. Observers were told that they were going to see one or two bright dots, one moving horizontally (the target) and the other moving vertically, diagonally, or horizontally in the opposite direction (the distractor). The observers' instruction was to always continue following the original horizontal spot and to ignore the distractor. Trials started with a small black fixation spot $\left(0.15^{\circ} \times 0.15^{\circ}\right)$ presented in the center of the monitor. Observers $(N=14)$ initiated each trial by pressing an assigned button on a game pad. When the drift correction was successful, the fixation dot disappeared. The target then appeared to the left or to the right of fixation at $5^{\circ}$ eccentricity along the horizontal meridian, and observers were required to make a saccade to the target. After $500 \mathrm{~ms}$, the target started to move toward the center of the screen at a constant velocity of $10.5 \%$ for approximately $1,000 \mathrm{~ms}$. At a random point in time, roughly at the center of the screen, the stimulus split into the target, which continued to move horizontally and which observers were supposed to track, and into the distractor stimulus with identical physical appearance that moved into one of eight possible directions: horizontally in the target direction $\left(0^{\circ}\right.$, baseline $)$, opposite to it $\left(180^{\circ}\right)$, or vertically $\left(90^{\circ}, 270^{\circ}\right)$ or diagonally $\left(45^{\circ}, 135^{\circ}, 225^{\circ}, 315^{\circ}\right)$ up or down. In the baseline condition, when target and distractor moved horizontally in the same direction, the physical appearance of the target was that of a single stimulus. Because there was no difference in tracking a leftward or rightward moving target in any of the experiments for any observer, we collapsed data for the two horizontal target directions here and in all other experiments. With two target directions (left, right) collapsed into one and eight distractor directions, Experiment 1 tested eight conditions. Each observer completed 20 test trials before the start of the experiment and completed one session with 384 trials in blocks of 64, resulting in 48 trials per condition for each observer. One observer completed two sessions. Figure 1 depicts a schematic diagram for one trial.

Results and discussion. Figure 2A shows horizontal eye velocity data across all observers for the time interval 0-320 ms after the split for the four cardinal distractor directions. The distractor stimulus had a strong effect on horizontal eye velocity: The eyes slowed down substantially by $25 \%$ (compared with eye velocity in the control condition) to a minimum of $7.8 \% \mathrm{~s}(S D=1.9)$ for distractors moving horizontally $\left(180^{\circ}\right)$, or diagonally $\left(135^{\circ}, 225^{\circ}\right)$ into the opposite direction as the target. The effect was less pronounced for vertical distractor directions and for distractors moving diagonally in the same direction as the target: Here, horizontal eye velocity slowed down by about $20 \%$ for vertical distractors and by $13 \%$ for diagonal distractors. The effect on horizontal eye velocity in the time interval 120-160 ms after stimulus split clearly varied with distractor direction (Figure 2C). This was supported by a one-way repeated measures analysis of variance (ANOVA), with distractor direction as a variable, $F(7$, $91)=88.9, p<.001$.

In contrast to the prediction of vector averaging, the eye vertically curved away from the distractor, and vertical eye velocity was higher in the direction opposite to the distractor (Figure 2B). The change in vertical eye velocity also depended on distractor direction: There was no effect for horizontal distractor movement, a small effect for a distractor moving diagonally in the opposite direction to the target, and a strong effect for diagonal distractors moving in the same direction as the target. The effect was most pronounced for vertical distractor movement: Vertical eye velocity 


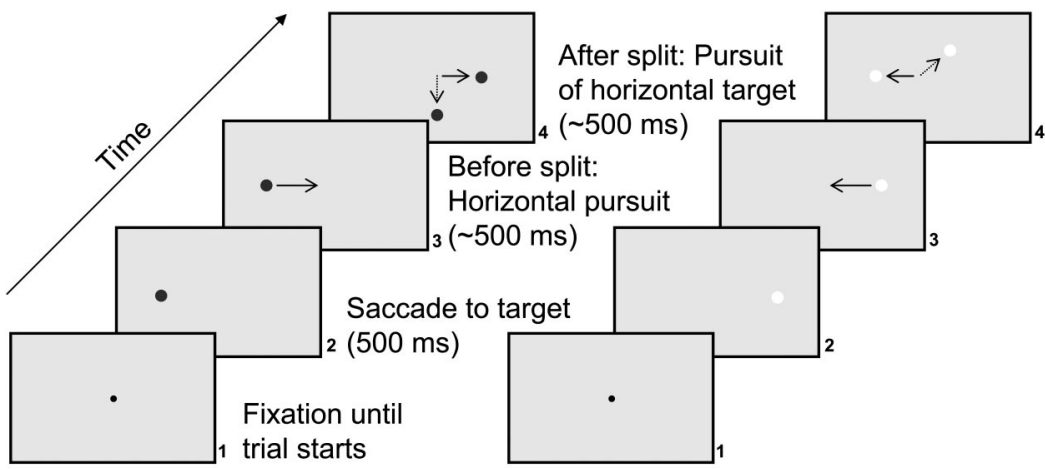

Figure 1. Sequence of events in a single trial in the basic experimental paradigm (Experiment 1). Each trial began with a fixation spot that was followed by a target step to the left or to the right of fixation position. While the target paused for $500 \mathrm{~ms}$, the observer had to make a saccade to it. The target then moved toward the center of the screen along the horizontal meridian and split into two stimuli at a random point in time, roughly at the center of the monitor. Observers were instructed to follow the horizontal target and ignore the distractor.

decreased $(-0.87 \% \mathrm{~s}, S D=1.4)$ for $90^{\circ}$ upward distractors and increased $(0.93 \%, S D=1.7)$ for $270^{\circ}$ downward distractors (Figure 2D). Vertical eye velocity significantly varied with distractor direction, as shown by a one-way repeated measures ANOVA, $F(7,91)=58.9, p<.001$

To further characterize the effect observed in the vertical velocity records, we looked at eye velocity traces for each individual observer averaged across trials within one condition. Out of 14 observers, 2 subjects ( 1 of them naive) had very noisy records and did not show a clear deviation of vertical eye velocity opposite to the distractor direction. For the remaining 12 observers, the effect was similar in shape and latency to the averaged data. Figure 3 shows mean vertical eye velocity traces for 4 selected and representative observers, 2 of whom were naive and 2 of whom were nonnaive. Latency of the effect was approximately $130 \mathrm{~ms}$, peak velocity varied between $1^{\circ}$ and $3 \%$, and duration of the effect was
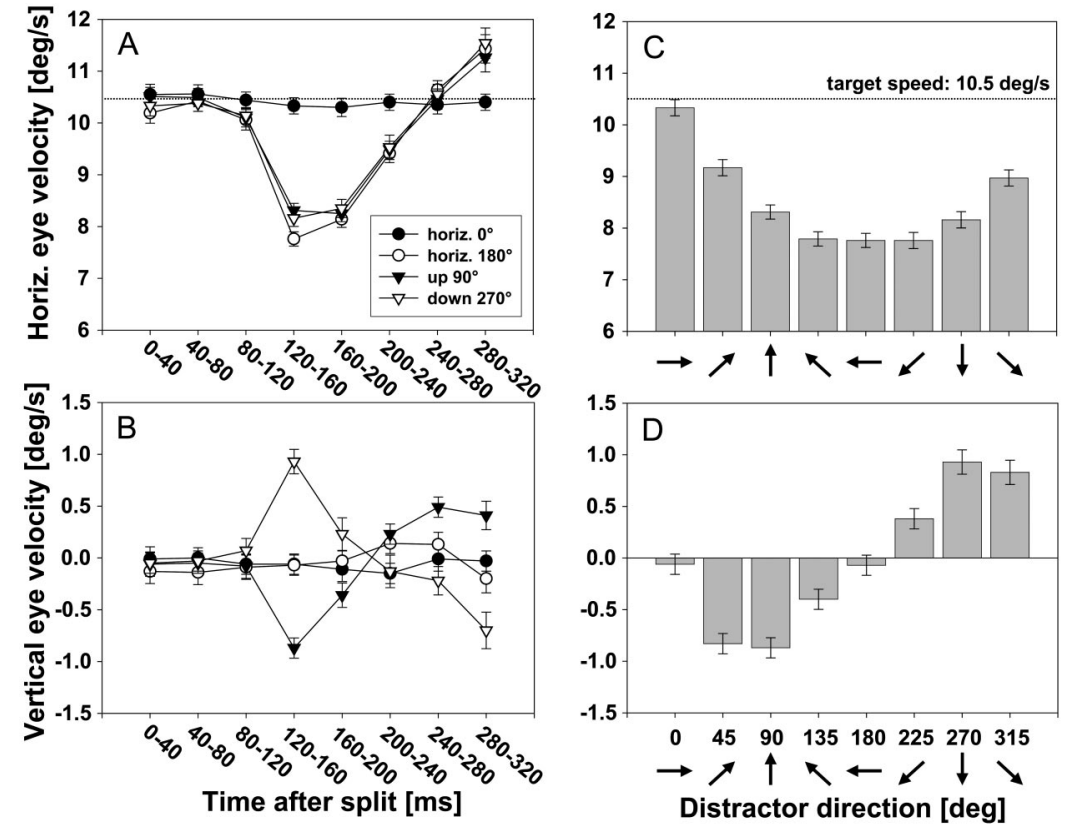

Figure 2. Mean horizontal and vertical eye velocity for tracking a horizontal target in Experiment $1(N=14)$. The error bars represent the $95 \%$ confidence intervals of the means $(S E=1.96)$. A: Horizontal eye velocity for cardinal distractor directions for time interval 0-320 ms after stimulus split. Solid circles denote the control condition, in which the distractor moved horizontally in the exact same direction as target. The dashed lines in Panels A and C denote target velocity $(10.5 \%)$. B: Vertical eye velocity for cardinal distractor directions for time interval $0-320 \mathrm{~ms}$. C: Horizontal eye velocity for all distractor directions averaged across time interval 120-160 ms after stimulus split. D: Vertical eye velocity for all distractor directions averaged across time interval $120-160 \mathrm{~ms}$. 

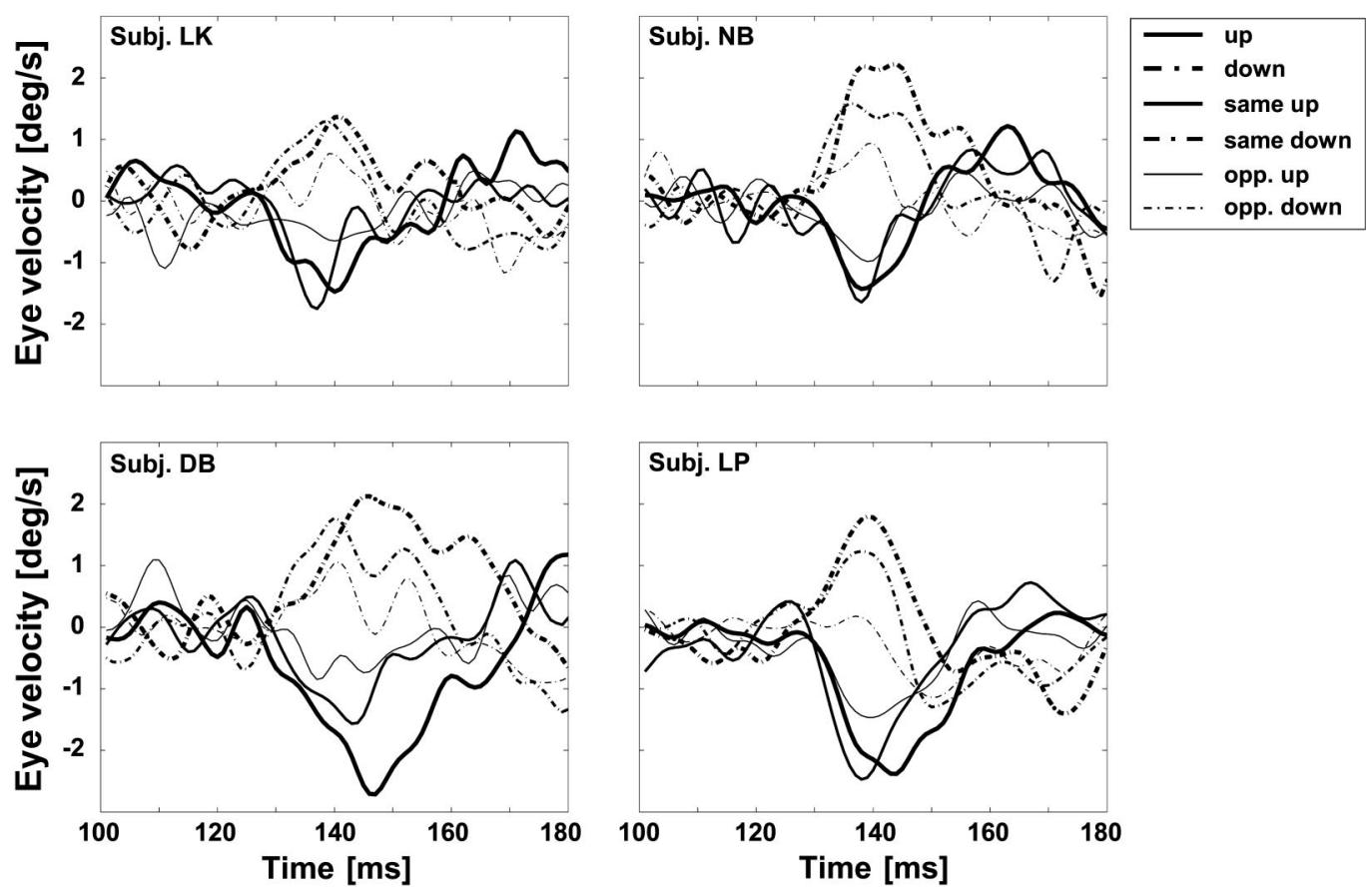

Figure 3. Mean vertical eye velocity for single observers averaged across trials within one condition. Traces were filtered by a Butterworth filter with a $60-\mathrm{Hz}$ cut-off. Different line types denote distractor directions. Time is given in ms after the split of the two stimuli (the split occurs at Time 0). Subjects (Subj.) LK and NB (top) were naive, whereas subjects DB and LP (bottom) were nonnaive.

approximately 20-40 ms. The main portion of the vertical deviation was often followed by an eye movement in the opposite direction, toward the direction of the distractor.

In addition, 3 observers showed a strong asymmetry in their vertical eye velocity response: When horizontal smooth pursuit eye movements were vertically deflected, the upward motion was faster than the downward motion. Because these 3 observers were used in all experiments, the up-down asymmetry can also be observed in the averaged data in Experiments 4-9 but not in Experiments 2 and 3. Similar asymmetries in vertical pursuit have been demonstrated by Lindner, Schwarz, and Ilg (2001). These authors showed that a brief shift of a structured background during steady-state tracking of a small target led to a stronger modulation of pursuit velocity when the background was shifted in phase (vs. counterphase) or upward (vs. downward). It is interesting to note that the vertical deviation of pursuit velocity was in the direction of the background shift. There is also evidence for asymmetries in vertical optokinetic nystagmus (OKN), showing that upward optokinetic stimulation elicits higher OKN gain than does downward motion (Lott \& Post, 1993). Further, vertical asymmetries have been reported in the monkey saccadic system (Zhou \& King, 2002). These authors claim that attentional sensitivity to visual targets is higher in the upper visual hemifield and that upward saccades are therefore faster, more accurate, and of shorter latency. The asymmetry in our results is not sufficiently consistent across observers to allow for further speculation.

The effect of vertical deviation away from the distractor was not restricted to horizontal target and vertical distractor motion. We showed this in a control experiment for vertical target direction with 4 observers ( 3 of whom were naive) that produced a clear effect in Experiment 1. With two vertical target directions, up or down, and two horizontal distractor directions, left or right, the vertical deviation effect was nearly identical in size to the one reported above. For a distractor going right the eye curved to the left with a mean horizontal velocity of $1 \%(S D=1.3)$ and for leftward distractor movement the eye curved rightward $(1 \% \mathrm{~s}$, $S D=1.1)$.

To summarize the results in Experiment 1: First, there was a large and very systematic detrimental effect of distractor motion on horizontal eye velocity. In the presence of a moving distractor, horizontal eye velocity decreased by $13 \%$ to $25 \%$, depending on the direction of the distractor. The horizontal component of the eye velocity therefore followed the vector average of the response evoked by the target and the distractor. Second, the eye vertically curved away from the distractor, and eye velocity therefore rose substantially in the direction opposite to the distractor by $1 \%$ s. The vertical component of the eye velocity did not follow the vector average.

Although the distractor had a substantial effect on eye velocity, the effect on eye position was very small (3 min/arc). Apparently, the purpose of the vertical deviation was not a substantial contribution in terms of getting the eyes anywhere.

\section{Experiment 2: Effect of a Vertical Distractor on Pursuit Initiation}

In the conclusion of their study, Lisberger and Ferrera (1997) stated that it is very difficult to overcome vector averaging, even 
when the tracking target is predefined by size or direction. We have shown that this is not the case during steady-state tracking of a predefined target. Lisberger and Ferrera further claimed that vector averaging is the computation that the pursuit system does naturally as a first response in the initiation phase of the eye movement. In Experiment 2 we tested whether the deviation effect was actually unique to steady-state tracking or whether the eye also curves away from the distractor during the initiation phase of pursuit.

Method. Observers $(N=5)$ initially fixated in the center of the screen. After starting the trial by pressing an assigned button, two stimuli appeared at the fixation point and instantly started to move in two directions, one moved horizontally (the target) and the other moved vertically, either up or down (the distractor). In four blocks of 96 trials each (1 observer did five blocks), observers were required to smoothly pursue the horizontal target as accurately as possible. We analyzed eye velocity in the same manner as before, but following the procedure described by Lisberger and Ferrera (1997), we now excluded all trials in which observers made an early saccade ( $<200 \mathrm{~ms}$ after target and distractor onset). Overall, $4.5 \%$ of all trials were excluded, leaving only trials in which the saccade occurred after $200 \mathrm{~ms}$.
Results. In this experiment, the eye movements were characterized by a brief, initial pursuit movement starting at about $80 \mathrm{~ms}$ after the stimulus onset. The initial pursuit was followed by a horizontal catch-up saccade at about $200 \mathrm{~ms}$ that brought the eye close to the target. Figure 4 shows the effect of an upward (Figure 4A) and a downward (Figure 4B) distractor, respectively, on horizontal and vertical eye velocity during pursuit initiation. The horizontal component of the pursuit eye movement showed an initial steady increase in velocity followed by a steep rise of velocity at about $200 \mathrm{~ms}$, therefore reflecting the pattern normally found in the initiation phase of a smooth pursuit eye movement (Rashbass, 1961).

It was surprising to note that the effect of the distractor on vertical eye velocity was similar in latency and duration to the one reported in Experiment 1, albeit smaller in amplitude and peak velocity, with a mean vertical velocity of $-0.48 \% \mathrm{~s}(S D=1.0)$ for upward and $0.54 \%$ s $(S D=1.1)$ for downward distractors. The effect of distractor direction was significant, as shown by a oneway repeated measures ANOVA, $F(1,4)=22.8, p=.009$. The
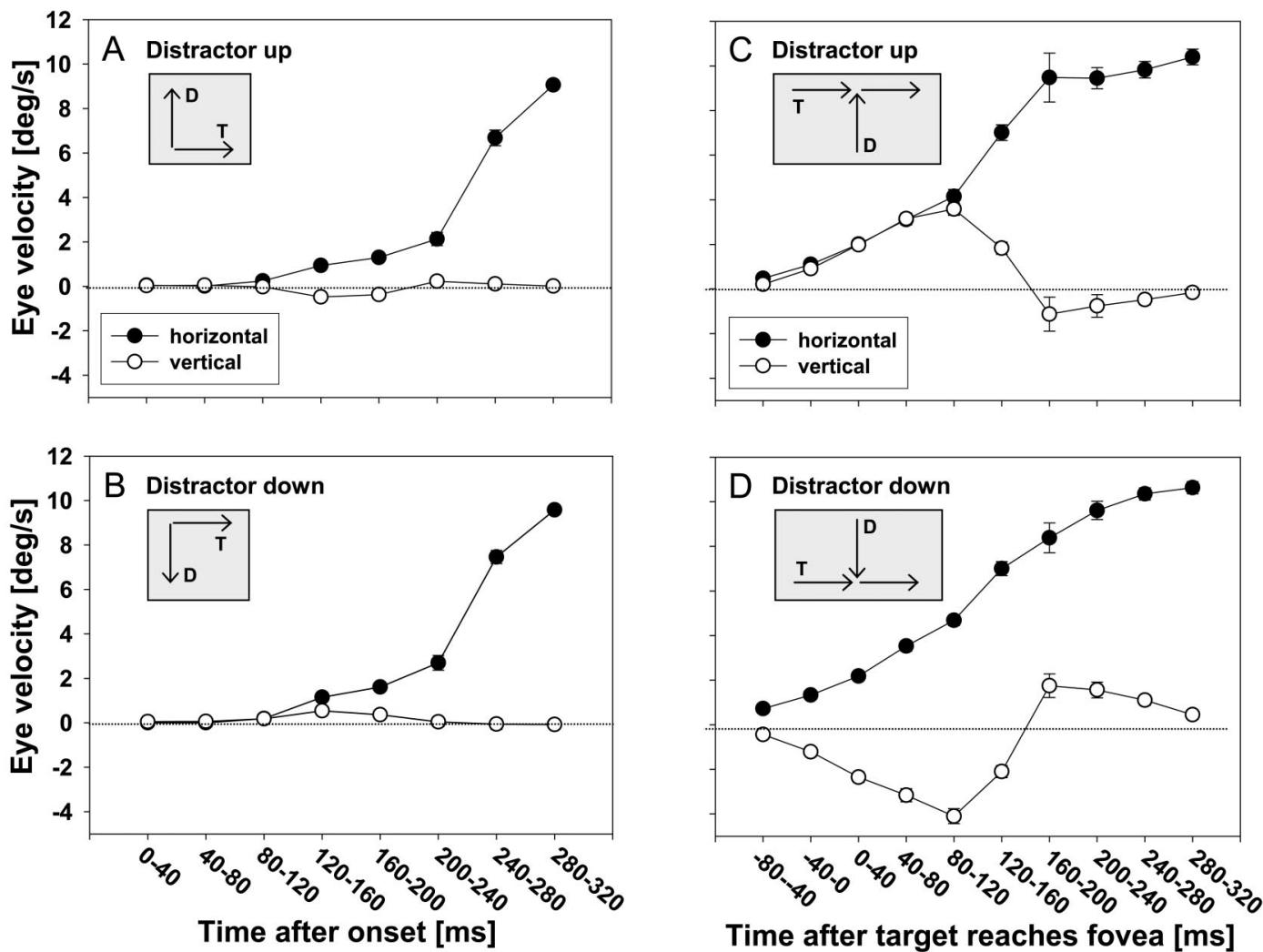

Time after target reaches fovea [ms]

Figure 4. A and B: Results for Experiment $2(N=5)$. Horizontal target (T) and vertical distractor (D) started to move at the same time from the center of the monitor after the fixation spot disappeared. A: Horizontal and vertical eye velocity for time interval $0-320 \mathrm{~ms}$ after the stimulus split for upward distractor motion. B: Horizontal and vertical eye velocity for time interval 0-320 ms for downward distractor. C and D: Results for replication of Lisberger and Ferrera (1997) experiment $(N=3)$. Horizontal target and vertical distractor started to move at the same time from the periphery toward fixation in the center of the monitor, and only one spot continued to move. Only selected results for horizontal target and up-down distractor directions are shown. C: Horizontal and vertical eye velocity for time interval $-80-320 \mathrm{~ms}$ after target reached fovea for upward distractor motion. D: Horizontal and vertical eye velocity for time interval $-80-320 \mathrm{~ms}$ for downward distractor. 
vertical deviation in Experiment 2 did not reveal an up-down asymmetry.

To recapitulate, the findings on vertical eye velocity that we have reported so far are neither in line with vector averaging nor with a winner-take-all model. The eye curved away from the distractor in a very systematic way and to a large extent both during steady-state tracking of a predefined horizontal target and during the initiation phase of a pursuit eye movement. However, it is important to note that our results in Experiment 2 are not directly comparable to those obtained by Lisberger and Ferrera (1997). In their study, the monkey fixated in the center of the screen while the target and distractor had already started to move toward the fixation cross. The monkey was supposed to start tracking the target as soon as it reached the fovea and when the distractor vanished. Most important, the monkey did not have any information about which of the two stimuli would become the target. In the experiments reported here, target and distractor started from the fovea and the observer had to pursue the target as soon as it appeared. Here, the observer always knew that the horizontally moving stimulus was the pursuit target. So far, we have shown that our findings are not restricted to the steady-state phase but also occur during pursuit initiation. But do these differences in results between our initiation experiments and that done by Lisberger and Ferrera (1997) rely on the fact that our observers had prior information, or is the effect due to other factors, such as differences in design or observers?

\section{Experiment 3: Replication of the Lisberger and Ferrera (1997) Experiment}

We next replicated the Lisberger and Ferrera (1997) experiment, which had originally been done on rhesus monkeys. In our version of the experiment, human observers $(N=3)$ initially fixated in the center of the monitor, while two stimuli moved toward the fovea from different directions. Target and distractor could move along one of the four cardinal axes. Once the two dots reached the fovea, only one stimulus (the target) moved on, whereas the distractor disappeared. The observer did not know which stimulus would become the target and in which of two possible directions it would move. We also presented trials with no distractor (baseline), resulting in 12 conditions (40 trials per condition). Selected results (for horizontal target and vertical distractor directions) are shown in Figure 4C (upward distractor) and Figure 4D (downward distractor). We were able to replicate the Lisberger and Ferrera (1997) findings for the equivalent experiment: The eye movement response followed the vector average of the two initial motion vectors, for example, when the distractor initially moved downward before disappearing, the eye movement initially had a downward component (see Figure 4D). Note that when the two spots reached the fovea, the eye already moved in the vector average direction with a velocity of $2 \%$. Fixation in 2 observers was very imprecise because anticipatory eye movements in the direction of the vector average occurred frequently (in approximately $30 \%$ of all trials). The effect peaked between 80 and $120 \mathrm{~ms}$ after the target reached the fovea and was considerably larger than the effect we found in Experiment 1. A one-way repeated measures ANOVA with distractor direction (up-down) as factor showed a significant effect, $F(1,2)=114.6, p=.009$

\section{Discussion and Summary of Part 1}

Figure 5 presents a summary of the main results obtained in Experiments 1-3. In Experiment 1, in which the distractor occurred during steady-state tracking and the observer had prior information about target motion, eye velocity went in the direction opposite to the vector average. If the vertical velocity component
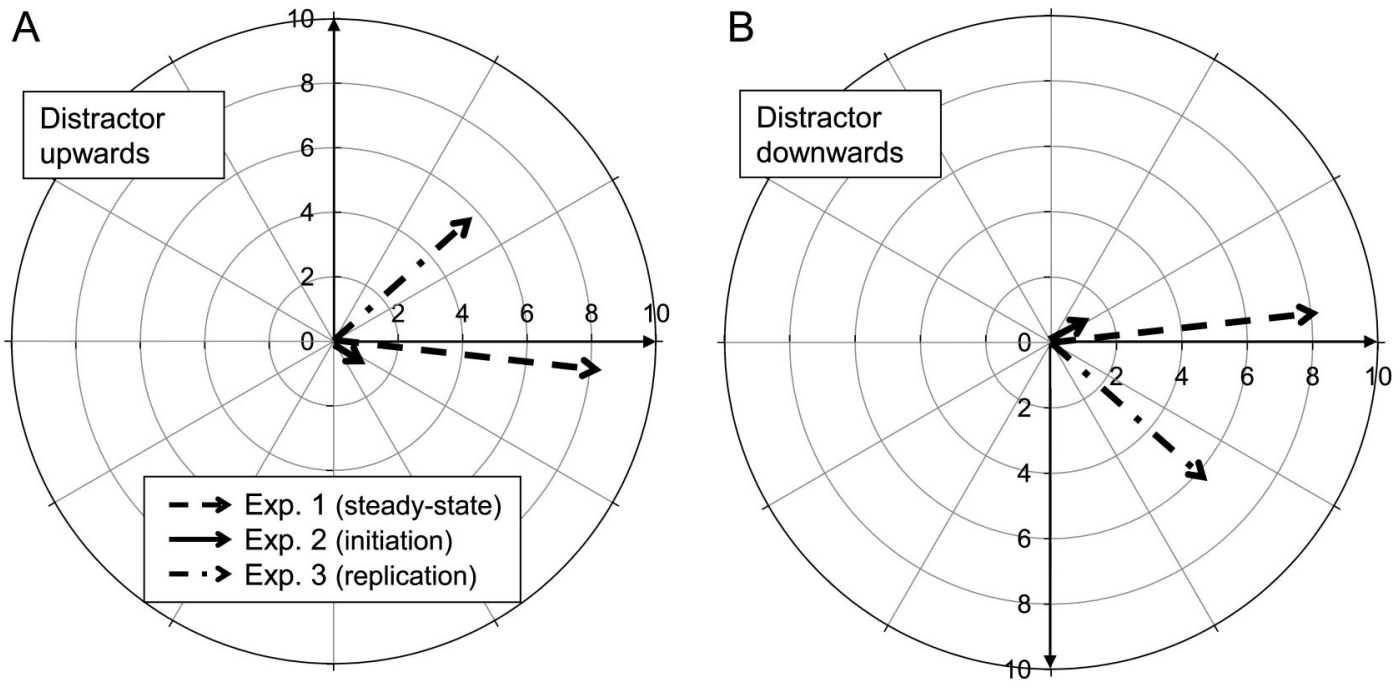

Figure 5. Polar plots summarizing results of Experiments 1-3 for horizontal target motion and vertical distractor motion. The direction of the velocity vectors indicates the direction of the eye movement; the length of the vectors indicates absolute velocity. A: Upward distractor motion. B: Downward distractor motion. In Experiment 1, eye velocity goes in the direction opposite to the vector average, but the vertical velocity component is much smaller than horizontal velocity. In Experiment 2, eye velocity goes exactly in the opposite direction of the vector average. In Experiment 3, eye velocity clearly follows the vector average. 
was inverted, the vector average model still would not be valid here because the vertical velocity component was only about one-tenth the size of the horizontal velocity. In Experiment 2, in which the distractor occurred during pursuit initiation and the observer had prior information about target motion, eye velocity went exactly in the opposite direction to the vector average. In Experiment 3, in which the distractor occurred during pursuit initiation and the observer had no information about target motion, eye velocity clearly followed the vector average.

\section{Part 2: Effect of Distractor Features on Pursuit Velocity}

The effect of a moving distractor on vertical velocity as found in Experiments 1 and 2 was very small, especially when compared to Experiment 3. A pursuit eye movement response of approximately the same size and duration has already been described by Wyatt, Pola, and Lustgarten (1989). The so-called "oculomotor twitch" has been reported to be a small, transient eye movement that occurs in response to the onset of target motion during both fixation and pursuit. It has a latency of approximately $100 \mathrm{~ms}$, a peak velocity of $1 \%$, and lasts for $50-100 \mathrm{~ms}$. This brief, seemingly automatic oculomotor response is therefore of similar latency and velocity as the effect on vertical eye velocity reported in Experiments 1 and 2. However, the oculomotor twitch differs from our results in a number of ways. First, the twitch goes in the direction of the target, whereas our effect goes in the direction opposite to a second moving stimulus. Second, twitch velocity varies with target direction (higher velocity for stimuli moving toward the fovea than away from it) but remains largely constant over a range of target velocities. We reported that the size of the effect of a moving distractor on vertical eye velocity varies systematically with distractor direction and is therefore highly sensitive to the physical properties of the stimulus. In the following experiments, we further test whether the deviation in the vertical eye movement response varies systematically with distractor features such as velocity (Experiment 4), contrast (Experiment 5), and number (Experiment 6).

\section{Experiment 4: Dependence on Distractor Velocity}

Method. Stimuli, paradigm, and task were identical to Experiment 1, except that target and distractor differed in velocity, and the distractor only moved into one of four directions: horizontally in the target direction $\left(0^{\circ}\right)$, opposite to the target $\left(180^{\circ}\right)$, or vertically $\left(90^{\circ}, 270^{\circ}\right)$ up or down. Distractor velocity was the same as the target's speed $\left(10.5^{\circ} / \mathrm{s}\right)$, slower $\left(3.5^{\circ} / \mathrm{s}\right)$, or faster $\left(17.5^{\circ} / \mathrm{s}\right)$. In the baseline condition $\left(0^{\circ}\right)$, the distractor always moved with the same velocity as the target, resulting in 10 conditions altogether. There were three times as many baseline trials than in the other conditions. After completing 20 test trials, observers $(N=6)$ did one or two sessions with 384 (768) trials in blocks of 96, resulting in 32 (64) trials per condition for each observer.

Results and discussion. The results for horizontal distractor direction (same or opposite as target) were similar to those in Experiment 1. Figure 6 shows the results for vertical distractor directions. Irrespective of its direction, the distractor had the same strong effect on horizontal eye velocity as in Experiment 1 (Figures 6A and 6C). A two-way repeated measures ANOVA with distractor direction (up, down) and velocity (same as target, slower, faster) did not show a statistically significant effect of direction, $F(1,5)=0.16, p=.71$. The decrease in horizontal eye velocity was numerically more pronounced for low than for high distractor velocity, but the main effect of velocity was not significant, $F(2,10)=1.30, p=.31$. The significant interaction, $F(2$, $10)=11.90, p=.002$, resulted from an up-down asymmetry in the effect of the distractor: Horizontal eye velocity decreased more for upward than for downward distractor motion, but only when target and distractor differed in velocity.

The vertical deviation opposite to the distractor was numerically less pronounced when the distractor moved slower than the target and more pronounced when distractor velocity was higher than target velocity (Figures 6B and 6D). A two-way repeated measures ANOVA (Distractor Direction $\times$ Velocity) yielded a significant main effect of direction, $F(1,5)=28.60, p=.003$, but not of velocity, $F(2,10)=0.42, p=.67$. The significant interaction, $F(2$, $10)=17.40, p=.001$, reflects the increase of the difference between upward and downward distractors for increasing distractor velocity. We further conducted separate one-way repeated measures ANOVAs for upward and downward distractor motion with distractor velocity as a factor. The effect of distractor velocity on vertical eye velocity was significant when the distractor was moving downward, $F(2,10)=10.24, p=.004$, but not when it was moving upward, $F(2,10)=3.93, p=.055$. We conclude that the effect of a vertical distractor on tracking a horizontal target scales with distractor velocity, in particular for downward distractor motion.

\section{Experiment 5: Contribution of Distractor Contrast}

It has been demonstrated in previous studies that motion perception and pursuit velocity strongly depend on contrast (Spering, Kerzel, Braun, Hawken, \& Gegenfurtner, 2005; Thompson, 1982). A higher distractor contrast and/or a lower target contrast produce higher motion energy in the distractor direction. Intuitively, one could assume that this might result in a smaller deviation effect. Alternatively, if the curvature effect found so far depended on suppression of distractor information, a more dominant distractor would necessitate a stronger suppression and therefore a larger deviation effect. Here, we examine whether the size and direction of the deviation effect depends on target or distractor contrast.

Method. In Experiment 5, we tested the effect of different target and distractor contrasts on 5 observers. One of the two stimuli, either the target or the distractor, was presented at a fixed luminance of $58 \mathrm{~cd} / \mathrm{m}^{2}$, whereas the luminance of the other stimulus was randomly sampled from a set of three values $\left(35,48,58 \mathrm{~cd} / \mathrm{m}^{2}\right)$, resulting in Weber contrasts of approximately $9 \%, 50 \%$, and $81 \%$. Distractors moved into one of two directions: vertically $\left(90^{\circ}\right)$ up or down. We expected the effect on vertical velocity to increase with increasing distractor contrast or decreasing target contrast.

Results and discussion. First, we report the results for a change in distractor contrast (see Figure 7). The effect of vertical distractors on horizontal eye velocity are shown in Figures 7A and 7C. Irrespective of distractor direction, there was an increase in horizontal slowing with increasing distractor contrast. Accordingly, a two-way repeated measures ANOVA (Distractor Direction $\times$ Contrast) for mean horizontal eye velocity at the relevant time interval after the split revealed a significant main effect of contrast, $F(2,8)=60.50, p<.001$, but not of distractor direction, $F(1,4)=0.60, p=.48$. The interaction also was nonsignificant $F(2,8)=1.10, p=.39$. 

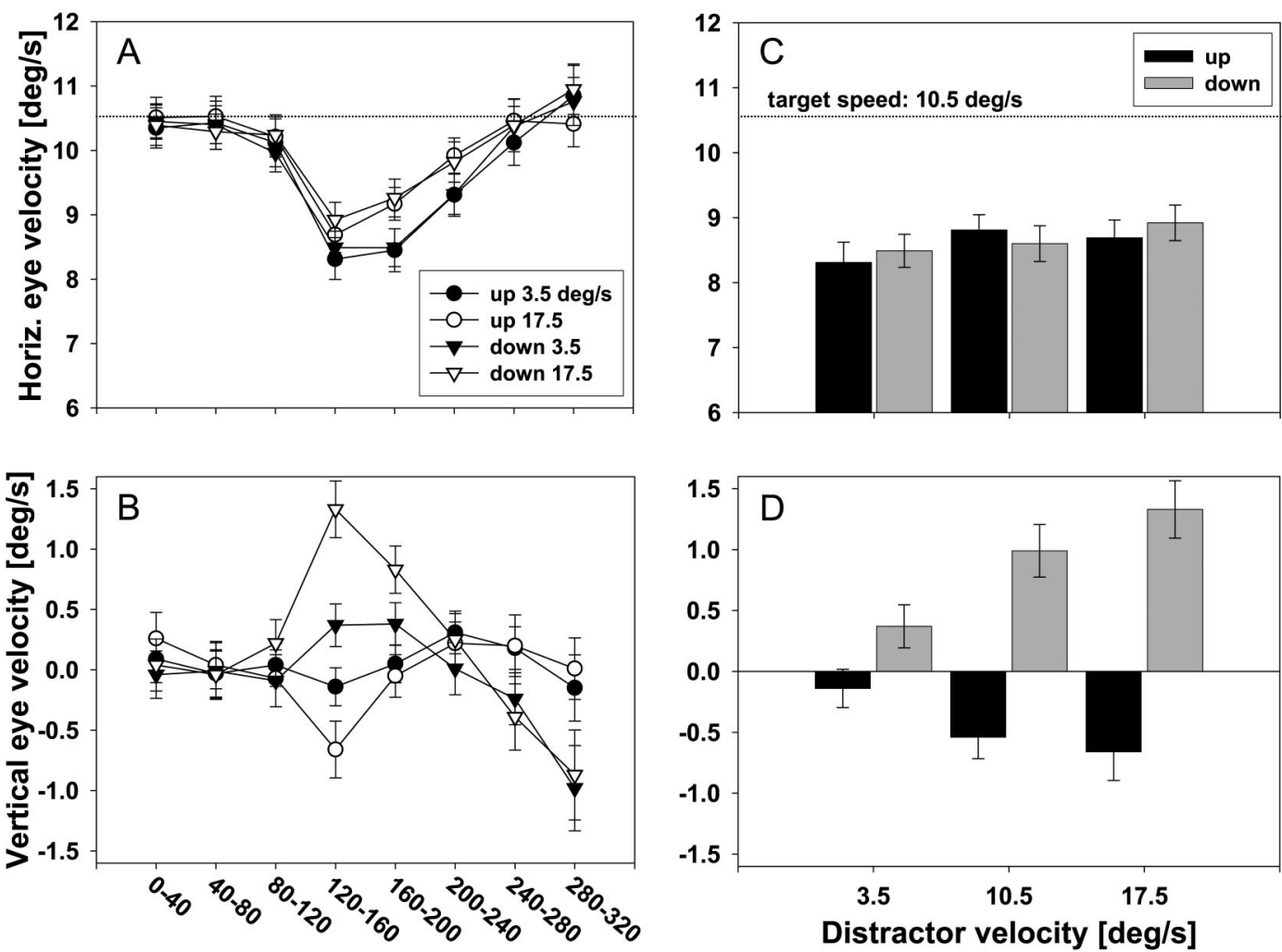

Time after split [ms]

Figure 6. Means and confidence intervals of horizontal and vertical eye velocity for tracking a horizontal target in Experiment $4(N=7)$. A: Horizontal eye velocity for time interval $0-320 \mathrm{~ms}$ for vertical distractor directions and two distractor velocities, which were slower $\left(3.5^{\circ} / \mathrm{s}\right)$ or faster than the target $\left(17.5^{\circ} / \mathrm{s}\right)$. B: Vertical eye velocity for time interval $0-320 \mathrm{~ms}$ for same conditions as in Panel A. C: Horizontal eye velocity for all distractor directions and velocities (including distractor velocity $10.5 \%$, same as the target), averaged across time interval $120-160 \mathrm{~ms}$. D: Vertical eye velocity averaged across time interval $120-160 \mathrm{~ms}$ for the same conditions as in Panel C.

Mean vertical deviation is plotted in Figures 7B and 7D. The pattern of the vertical deviation was similar to the results in our previous experiments, and a two-way repeated measures ANOVA (Distractor Direction $\times$ Contrast) showed a significant main effect of distractor direction, $F(1,4)=13.90, p=.02$. The deviation was larger for downward than for upward distractor motion. However, vertical deviation did not increase significantly with increasing distractor contrast, $F(2,8)=3.80, p=.07$. The significant interaction between distractor direction and contrast, $F(2,8)=$ $11.20, p=.005$, reflects the increase of the difference between upward and downward distractors with increasing distractor contrast. For low-contrast downward distractors, there was a downward deviation of the eye. This effect was very small but was significantly different from zero, $t(4)=-6.33, p=.003$. However, the effect for upward distractors at low contrast was not significantly different from zero, $t(4)=-2.31, p=.08$. Therefore, the vertical deviation effect nearly disappeared when the distractor was presented at low contrast.

For a change in target contrast, we expected to see a larger vertical deviation effect for a lower target contrast. Indeed, the results here corresponded to those for a change in distractor contrast (see Figure 8). However, for medium and low target contrast, the effect on horizontal and vertical velocity was very different in shape to the results reported so far. For high target contrast, the horizontal component of the eye velocity reached its peak minimum at the same time interval after the split than in the experiments before. For medium and low contrast, however, the effect peaked $40 \mathrm{~ms}$ later and was prolonged in duration. The effect on vertical eye velocity also had a longer recovery rate for medium and low target contrast. To summarize, there was a tendency for the effect to rise as the distractor signal strength increased (high distractor contrast or low target contrast).

\section{Experiment 6: Effect of a Second Vertical or Diagonal Distractor}

In the preceding experiment, we saw that an increase in distractor contrast increased the vertical deviation. With the following experiment, we aim at answering the question of whether the deviation effect also increases as we increase the number of distractors. Both effects would indicate that distractor signals are summed up and that the sum determines the size of the vertical deviation. We test this by introducing a second distractor into the visual field. We are particularly interested to see whether two 

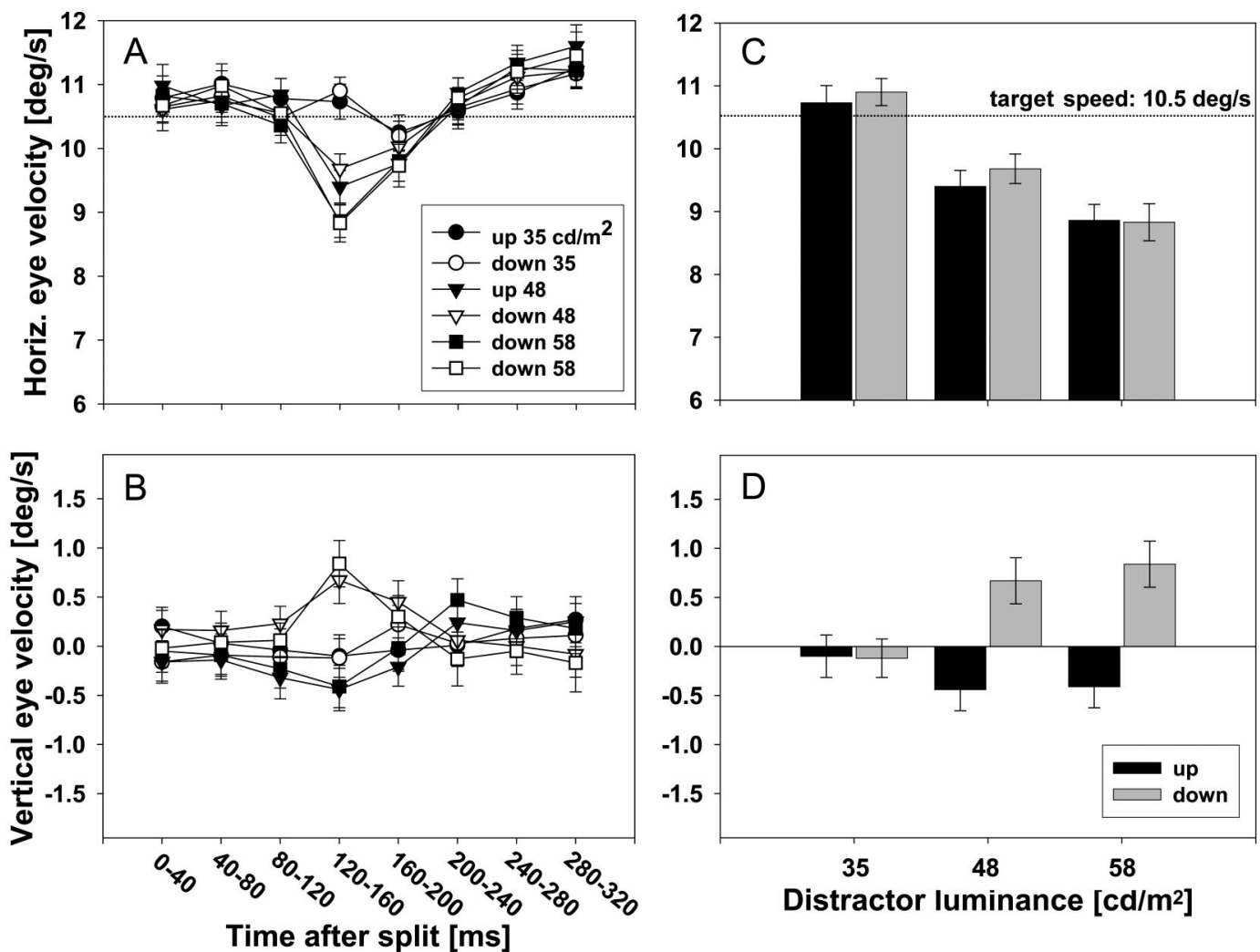

Figure 7. Means and confidence intervals of horizontal and vertical eye velocity for tracking a horizontal target in Experiment $5(N=5)$. The distractor varied in luminance. A: Horizontal eye velocity for time interval 0-320 $\mathrm{ms}$ for vertical distractor directions and three levels of distractor luminance. B: Vertical eye velocity for time interval 0-320 ms for same conditions as in A. C: Horizontal eye velocity for all distractor directions and levels of distractor luminance averaged across time interval $120-160 \mathrm{~ms}$. D: Vertical eye velocity averaged across time interval $120-160 \mathrm{~ms}$ for the same conditions as in Panel C. $\mathrm{cd} / \mathrm{m}^{2}=$ candelas $/ \mathrm{m}^{2}$.

distractors moving in vertically opposite directions cancel each other out, as spatial summation would predict.

Method. In this experiment, a vertical distractor was paired with a second diagonal distractor. There was also a condition in which two vertical distractors were shown, one moving vertically up and the other moving vertically down. In the baseline condition, there was no distractor. We tested six conditions on 8 observers, who did one session with 480 trials each.

Results. The effect of a second vertical or diagonal distractor on horizontal eye velocity is shown in Figures 9A and 9C. Concerning cases in which one distractor moved vertically and the second one moved diagonally, there was no difference between upward and downward distractor direction. The corresponding two-way repeated measures ANOVA on diagonal distractor direction (Up-Down Vertical $\times$ Same-Opposite) did not show a significant effect of up-down distractor direction, $F(1,8)=0.65$, $p=.44$. For diagonal distractors, the decrease in horizontal eye velocity was generally larger when the second distractor was moving diagonally into the opposite direction as the target. This effect of diagonal distractor direction was significant, $F(1,8)=$ 25.96, $p=.001$. There was no significant interaction, $F(1,8)=$ $1.68, p=.23$

Effects on vertical eye velocity are shown in Figures 9B and 9D. When two distractors (one vertical, one diagonal) were present, the effect of up-down distractor direction was similar to corresponding results from our previous experiments. A two-way repeated measures ANOVA with vertical and diagonal distractor direction as factors showed a significant effect of up-down distractor direction, $F(1,8)=32.90, p<.001$, but no effect of diagonal distractor direction, $F(1,8)=0.06, p=.82$. The interaction also was nonsignificant, $F(1,8)=2.50, p=.15$. It is interesting to note that when two vertical distractors moved in opposite directions (up and down), there was no effect on vertical eye velocity, $t(8)=0.24, p=.82$.

Compared with results from Experiment 1, the effect of two distractors was not generally numerically larger than the effect of one vertical distractor: For horizontal eye velocity, the effect of two combined distractors moving into the same hemifield (up or down) was not numerically larger than the effect of the single distractor as in Experiment 1, with the exception of a combined upward and diagonally opposite distractor $(8.1 \%, S D=2.2)$, which produced a larger horizontal slowing than a single upward distractor as in Experiment $1\left(8.3^{\circ} \mathrm{s}, S D=1.8\right)$. For vertical eye velocity, the effect was only numerically larger for two distractors moving downward in the same hemifield direction $(1.03 \%$ s, $S D=$ 0.8) compared with a downward vertical distractor as in Experiment $1\left(0.93^{\circ} \mathrm{deg} / \mathrm{s}, S D=1.7\right)$. However, it is difficult to directly 

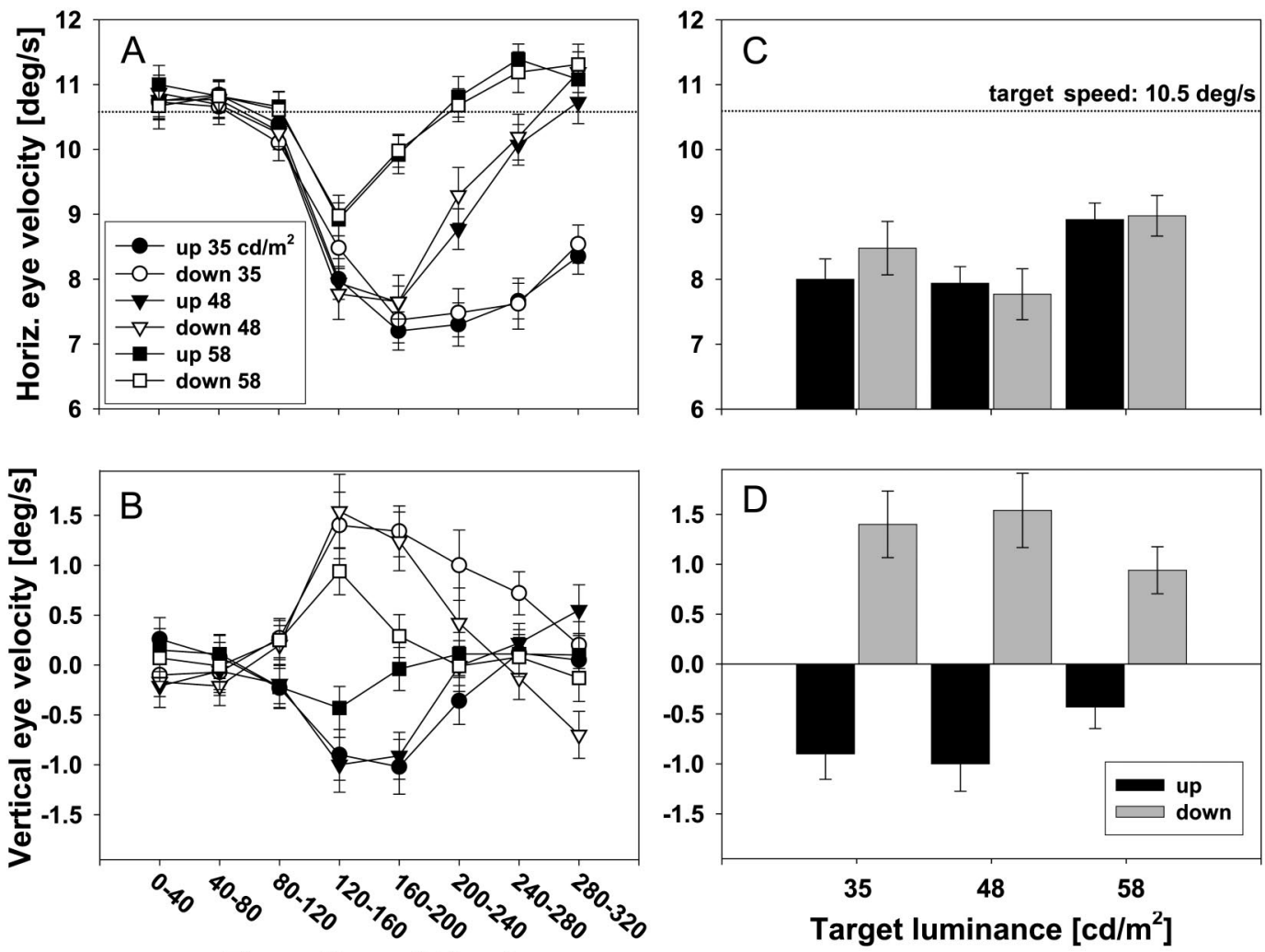

Time after split [ms]

Figure 8. Means and confidence intervals of horizontal and vertical eye velocity for tracking a horizontal target with different luminance in Experiment $4(N=5)$. A: Horizontal eye velocity for time interval $0-320 \mathrm{~ms}$ for vertical distractor directions and three levels of target luminance. B: Vertical eye velocity for time interval 0-320 $\mathrm{ms}$ for the same conditions as in Panel A. C: Horizontal eye velocity for all distractor directions and levels of target luminance averaged across time interval $120-160 \mathrm{~ms}$. D: Vertical eye velocity averaged across time interval $120-160 \mathrm{~ms}$ for same conditions as in Panel C. $\mathrm{cd} / \mathrm{m}^{2}=$ candelas $/ \mathrm{m}^{2}$.

compare results from those two experiments because of possible differences between observers (e.g., see discussion of up-down asymmetries above).

To summarize, we found that a second diagonal distractor did not add considerably either to the horizontal slowing effect or to the vertical deviation effect obtained for one vertical distractor. When both distractors moved into vertically opposite directions, the vertical deviation effect was canceled.

\section{Discussion and Summary of Part 2}

So far, we have shown that the vertical deviation effect varied with direction, velocity, and contrast in a systematic way. A second distractor moving vertically into the opposite direction to the first distractor canceled the effect of vertical deviation. We can rule out the possibility that the effect is simply an oculomotor reflex that occurs in response to the onset of distractor motion. Whereas the oculomotor twitch seems to be a feature of the output system, we are confident that the highly systematic effect found here reflects the physical properties of the stimulus. In the following two parts, we test different assumptions that might explain the origins of the effect.

\section{Part 3: Testing the Contribution of Misperception}

The first assumption is that the motor deviation opposite to the distractor is the result of a perceptual misinterpretation of either target or distractor motion trajectory. In order to test this assumption, we must first show that the deviation effect we found in the motor response extends to the perceptual system. Perceptual errors in localizing stationary or moving objects during smooth pursuit eye movements are well documented (Schlag \& Schlag-Rey, 2002). When observers have to judge the perceived starting position of a moving target, they make localization errors both in the direction of motion (Fröhlich effect; Fröhlich, 1923) and opposite from it (onset repulsion effect; Thornton, 2002). The direction of mislocalization seems to depend on stimulus context: When the stimulus appeared at predictable positions, pointing errors were displaced in motion direction; when onset position was unpredictable, the displacement was in the opposite direction (Kerzel \& Gegenfurtner, 2004; Müsseler \& Kerzel, 2004). The mislocalization effect in both directions also scales with stimulus velocity. In our experiments, distractor starting position was unpredictable along the horizontal axis. Accordingly, we expect the localization 

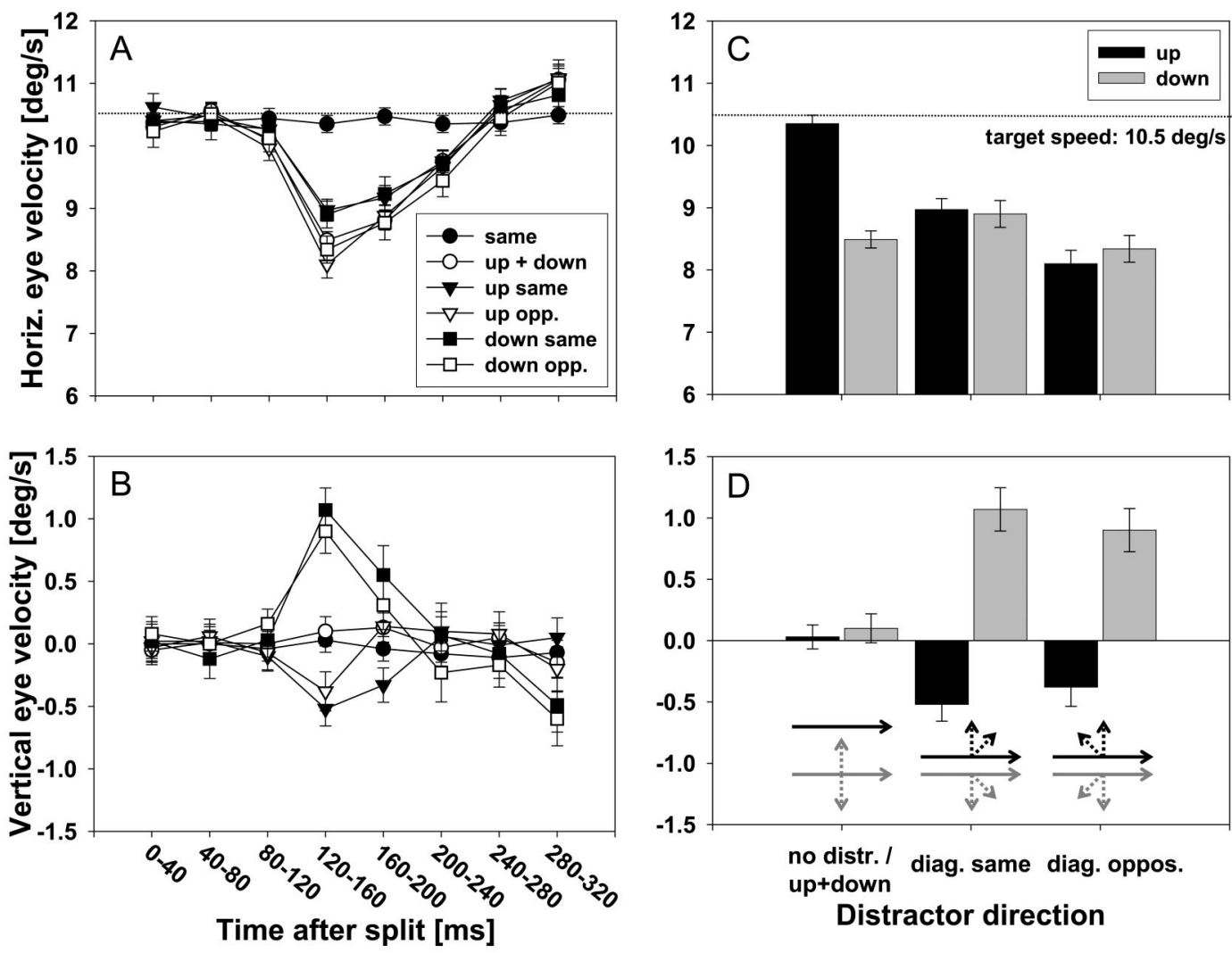

Figure 9. Means and confidence intervals of horizontal and vertical eye velocity for tracking a horizontal target in the presence of two distractors in Experiment $6(N=8)$. A: Horizontal eye velocity for time interval 0-320 $\mathrm{ms}$ for no distractor (baseline), for two distractors moving in opposite vertical directions, or for distractors moving vertically and diagonally up or down. B: Vertical eye velocity for time interval $0-320 \mathrm{~ms}$ for the same conditions as in Panel A. C: Horizontal eye velocity for all directions for two distractors averaged across time interval 120-160 ms. D: Vertical eye velocity averaged across time interval 120-160 ms for the same conditions as in Panel C.

of onset position to be shifted in the direction opposite from distractor motion.

Alternatively, the target trajectory could be perceived to be curved in the direction opposite from distractor direction. However, none of the observers reported any perception of curvature in the target trajectory. Nonnaive observers knew that the target trajectory was a straight line, and still showed the effect. Therefore, we varied the starting position of the distractor in Experiment 7.

\section{Experiment 7: Is Vertical Deviation Caused by Misperception at Distractor Onset?}

Method. In addition to following the horizontal target, observers $(N=$ 7) had to indicate the starting position of the vertical distractor. The basic paradigm for this experiment was the same as for Experiment 1. The target moved leftward or rightward, and the distractor moved either vertically up or down. The distractor starting position was either on the target trajectory (control condition; same as in experiments before) or one, two, four, or eight pixels above or below with regard to the target trajectory. Observers had to indicate whether the distractor had started above or below the trajectory, irrespective of its direction of movement, by pressing assigned arrow keys on the keyboard. If the perceptual judgment is in line with the motor response, it should be shifted in the opposite direction of distractor motion. A distractor starting on the target trajectory (control) would therefore be perceived to start below the trajectory. Three observers did one session with 20 conditions (2 Distractor Directions $\times 10$ Onset Positions). Two observers did one session with starting positions varying between four pixels above or below the target trajectory only, therefore resulting in 16 conditions, and 2 observers completed both sessions. Observers completed between 400 and 768 trials.

Results and discussion. Figure 10A depicts the psychometric functions for responses to starting positions for both distractor directions. A negative value for relative start position denotes conditions in which the distractor crossed the target trajectory. Here, the start position was shifted back along the trajectory of the distractor. A relative starting position of zero denotes the baseline conditions, in which distractors started on the trajectory of the target. Positive relative start position values stand for conditions in which the distractor started above (for upward distractors) or below (for downward distractors) the trajectory of the target.

For both distractor directions, psychometric functions were shifted leftward and judgments were biased in the direction of the distractor movement. The results are not in line with our expectations, and the perceptual judgment is in contradiction to the motor 

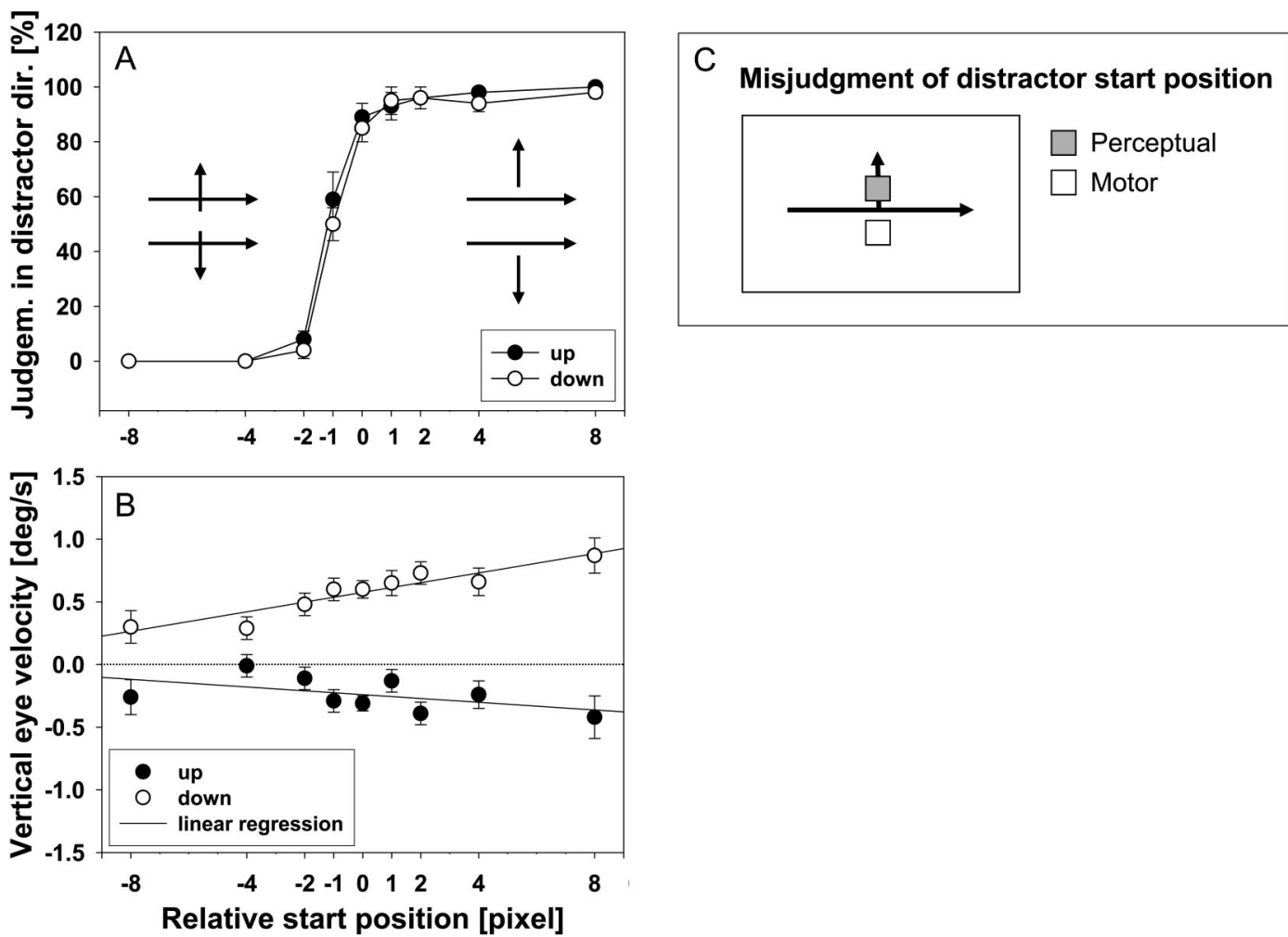

Figure 10. Results for Experiment $7(N=7)$. A: Judgments of the starting position of a vertical distractor moving up or down (in percentages) in distractor direction. B: Vertical eye velocity averaged for time interval $120-160 \mathrm{~ms}$ for nine starting positions for upward and downward distractor movement. Larger error bars in the two 8-pixel conditions result from a smaller number of trials. The solid lines through the vertical velocity data points are linear regression lines. C: Summary of results. The perceptual judgment is biased in the direction of the distractor motion; the motor response goes in the direction opposite to the distractor.

response of the eye. Figure 10B shows mean vertical eye velocity in the relevant time interval for both distractor directions and nine distractor starting positions. Visual inspection of the data shows that the vertical deviation in the direction opposite from the distractor (and therefore opposite from the perceptual judgment) had a tendency to increase as starting position moved down the distractor trajectory into the movement direction. We used linear regression analysis, conducted separately for the two distractor directions, to quantify the influence of starting position as an independent variable on vertical eye velocity as a dependent variable. The slope of the linear regression was significantly different from zero for downward distractor and upward eye movement, $t(5)=5.53, p=.001$, but not for upward moving distractors, $t(5)=-1.87, p=.10$.

Therefore, the vertical velocity deviation away from the distractor increased with increasing distance of distractor onset position from target trajectory for downward distractors but not for upward moving distractors.

As summarized in Figure 10C, observers tend to place the distractor starting position forward in the direction of motion, and perception therefore follows the classical Fröhlich effect. The eye movement, on the other hand, is deviated in the opposite motion direction. Although the motor response tended to deviate away from the distractor, the visual response deviated toward the dis- tractor. We conclude that the effect of vertical deviation cannot be caused by a perceptual misperception of the distractor starting position.

\section{Experiment 8: Contribution of a Static Reference Frame}

Experiment 7 ruled out the possibility that the vertical deviation effect resulted from a perceptual localization error with regard to the distractor position at the split. However, it is still possible that the horizontal target is perceived to have an illusory vertical component because the vertical distractor might be used as a reference.

Method. In Experiment 8, we therefore used a static background as a reference system to facilitate the identification of target and distractor at the time of split. If observers $(N=3)$ falsely perceive the target to have a vertical component in its smooth horizontal trajectory, a reference frame should diminish the effect of vertical deviation.

Stimuli, paradigm, and task were identical to Experiment 1, but we only used the two vertical distractor directions. We introduced thin black vertical lines above and below the target trajectory as a static reference frame for the horizontal target. The reference frame consisted of varying numbers of lines: We either used a small reference frame with lines at $2.4^{\circ}$ length spaced at $1.4^{\circ}$. The target ran in a "tunnel" of $1.8^{\circ}$ width. Or we used a large reference frame that filled the entire screen, with lines spaced at $0.8^{\circ}$, and a tunnel of $1.1^{\circ}$ width (see Figure 11E) 

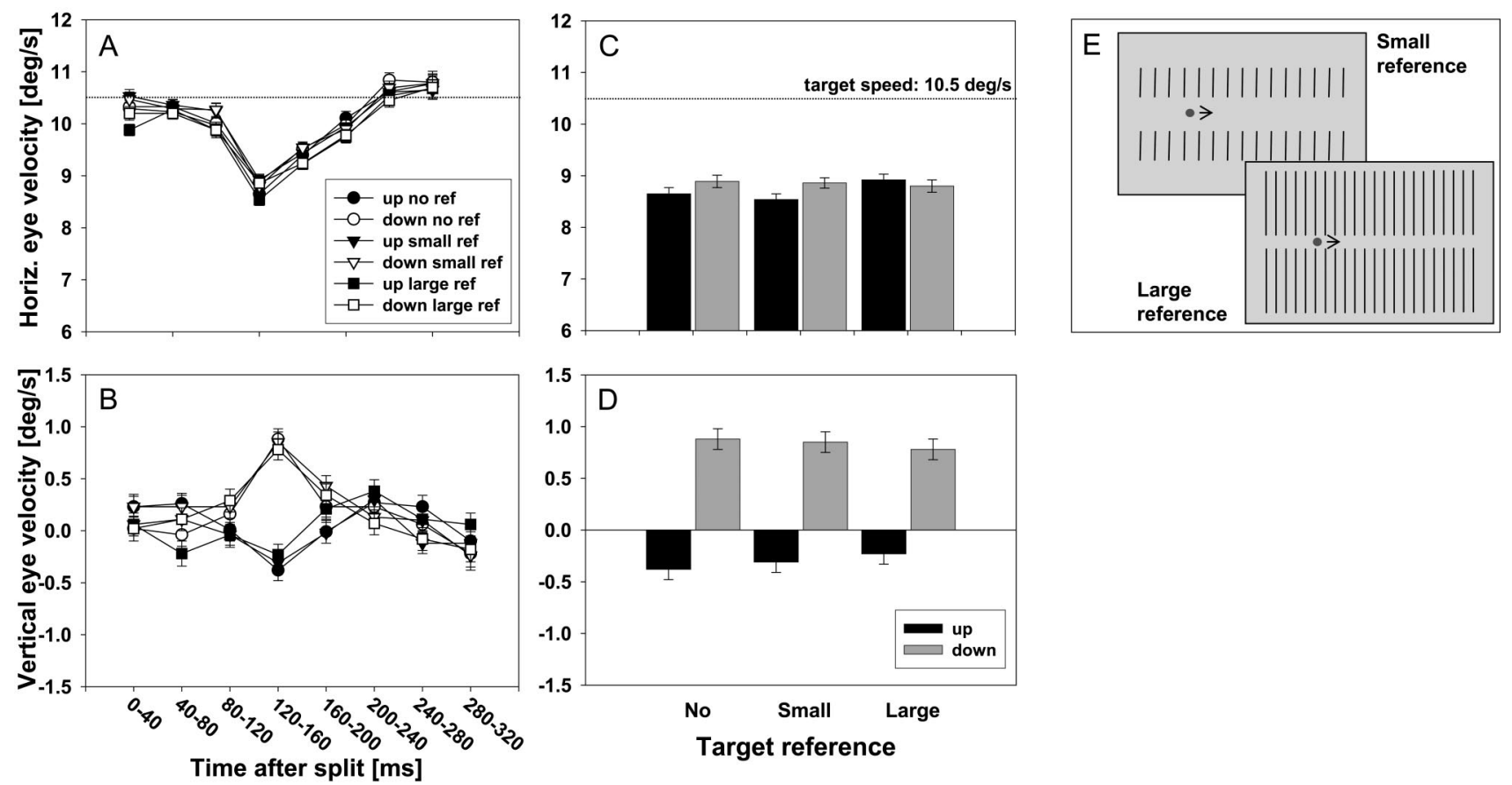

Figure 11. Means and confidence intervals of horizontal and vertical eye velocity for tracking a horizontal target in Experiment $8(N=3)$. The target was tracked in the presence of a small or large vertical reference frame or without a reference (control condition). A: Horizontal eye velocity for time interval $0-320 \mathrm{~ms}$ and for two vertical distractor directions. B: Vertical eye velocity for time interval $0-320 \mathrm{~ms}$ for the same conditions as in Panel A. C: Horizontal eye velocity averaged across time interval 120-160 ms. D: Vertical eye velocity for the same time interval as in Panel C. E: Schematic diagram for vertical reference frames used in the experiment.

In a preliminary version of this experiment, we used the small reference frame only without a baseline condition on a larger number of observers $(N=6)$. The presence of a vertical reference frame led to a decrease in the effect of distractor direction on vertical eye velocity; however, we were not able to directly compare our results with the data from Experiment 1. Therefore, we now introduced a baseline condition with no reference in this experiment, resulting in six conditions that were presented randomly interleaved.

Results and discussion. Figure 11 shows the effect of vertically moving distractors on horizontal (Figures 11A and 11C) and vertical eye velocity (Figure 11B and 11D). The effect on horizontal eye velocity was smaller than in Experiment 1 and did not vary significantly with distractor direction or reference frame condition. A two-way repeated measures ANOVA (Distractor Direction $\times$ Reference Frame Condition) did not show a significant main effect of direction, $F(1,2)=4.52, p=.17$, or of reference frame condition, $F(2,4)=1.00, p=.44$. The interaction was significant with $F(2,4)=8.05, p=.04$, and was due to differences in the effect of upward or downward moving distractors for different reference frames (see Figure 11C). For vertical eye velocity, the results found here showed a similar tendency as those in Experiment 1, and the same up-down asymmetry as in Experiments 4-7. A two-way repeated measures ANOVA showed that the effect of distractor direction was significant, $F(1,2)=$ $18.83, p=.049$. There was no effect of reference frame size, $F(2$, $4)=0.10, p=.90$. The interaction also was nonsignificant, $F(2$, 4) $=3.80, p=.12$.

The presence of a static reference did not prevent the eye from slowing down substantially in the horizontal domain. The eye still curved away from the distractor. We have to conclude from this experiment that the vertical deviation opposite from the distractor can only be due in part, if at all, to a misperception of the moving object's identity at the split.

\section{Discussion and Summary of Part 3}

In Experiments 7 and 8, we have shown that the vertical deviation effect does not result from a misperception of distractor onset position and is unlikely to result from a perceived perturbation of the target trajectory. We claim that the effect is not purely visual or the product of mechanisms such as induced motion in the direction opposite to the distractor. Rather, the effect might reflect the overcompensation for, or the suppression of, an automatic motor response to the distractor.

\section{Part 4: Testing the Contributions of Spatial Attention and Distractor Predictability}

As a second set of assumptions, we test the possible modulating role of cognitive factors, such as spatial attention and expectation. It is a classic finding in psychology that the sudden onset of a stimulus evokes an involuntary orienting response (Posner, 1980). However, an orienting response toward the distractor is not what we find here. On the contrary, the eye curves away from the distractor. Our results so far are in line with studies showing that saccades to visual targets also curve away from visual distractors to which the observer had previously oriented attention (Sheliga et 
al., 1994). In their premotor theory of attention, Rizzolatti et al. (1987) proposed that spatial attention is closely related to motor programming. In the case of attention, a motor plan is set but not executed; whereas in the case of action, the motor plan is set and executed. It is possible that the abrupt occurrence of the distractor in our experiments produced a covert shift of attention followed by a movement plan into the direction of the distractor. Because of the verbal instruction to ignore the distractor, the motor plan is inhibited and the movement not executed. The vertical deviation might then result from an overcompensation of the movement plan toward the distractor.

In the next experiment, we therefore address the question of whether the effect is modulated by spatial attention. We used a partially valid cuing paradigm to direct observers' spatial attention to either the target or the distractor. If the vertical deviation is modulated by spatial orientation, the effect should be larger when attention is directed toward the distractor because more effort has to be made to inhibit the movement plan toward the distractor.

\section{Experiment 9: Is the Distractor Effect Modulated by Spatial Attention?}

Method. We used a partially valid (80/20) cuing paradigm to test whether voluntary spatial attention to the distractor has an influence on the effect. While tracking a horizontal target, observers $(N=12)$ had to detect a brief, 100-ms luminance decrement that occurred $150 \mathrm{~ms}$ after the split in either the horizontal target or the vertical distractor (see Figure 12). After each trial, observers had to judge whether the luminance had changed by pressing assigned keys on the keyboard. They were not supposed to judge where the luminance change had occurred but only whether it had occurred at all. The size of the luminance decrement necessary for each observer to detect the change was determined preliminarily to the main experiment. We used a standard one-up two-down double-interleaved staircase procedure converging at $71 \%$ correct. The procedure ended automatically after six reversals were reached for each staircase (approximately 50 trials). On average, observers needed an $18 \%$ luminance decrement for changes in the target and a $52 \%$ luminance decrement for changes in the distractor.

Each trial started with a central endogenous cue in the shape of a triangle that was pointed to either the target or the distractor. The cue served as the fixation object and was valid in $80 \%$ of the trials. When the luminance change occurs in the direction that was previously cued (a valid cue), detection performance should be fairly accurate ( $75 \%$ correct); whereas a luminance change occurring in the noncued direction (an invalid cue) supposedly yields a detection performance at chance level (50\% correct). Performance later served as a control of whether the triggering of attention was successful. Eye movements were only analyzed in those trials where luminance did not change (50\%).

Results and discussion. Initially, we calculated the proportion correct for performance in detecting the contrast increment in valid and invalid trials. As expected, proportion correct across all observers was higher in valid $(71 \%, S D=0.06)$ than in invalid $(63 \%$, $S D=0.05)$ trials. Arcsine-transformed percentage-correct data were used to compare the performance in valid and invalid trials by inferential statistical analysis. The transformed data yielded the same results in the ANOVA as the nontransformed data. The ANOVA revealed a significant difference, $F(1,22)=12.13, p=$ .002 . We can therefore assume that attention was shifted to the cued location (either the target or the distractor).

Pursuit characteristics were analyzed in trials without a contrast increment only so that the effects of contrast change and attention were not confounded. Figure 13 shows that vertical velocity deviated from the distractor to the same extent for the two cuing conditions. For distractors moving upward, mean peak eye velocity was $-0.41 \%(S D=0.28)$ for cued target direction and $-0.38 \%$ s $(S D=0.32)$ when the distractor was cued. For downward distractors, mean peak eye velocity for cued target direction was $0.86 \%$ s $(S D=0.59)$ and for cued distractor direction $0.71 \%$ $(S D=0.31)$. A two-way repeated measures ANOVA (Cuing

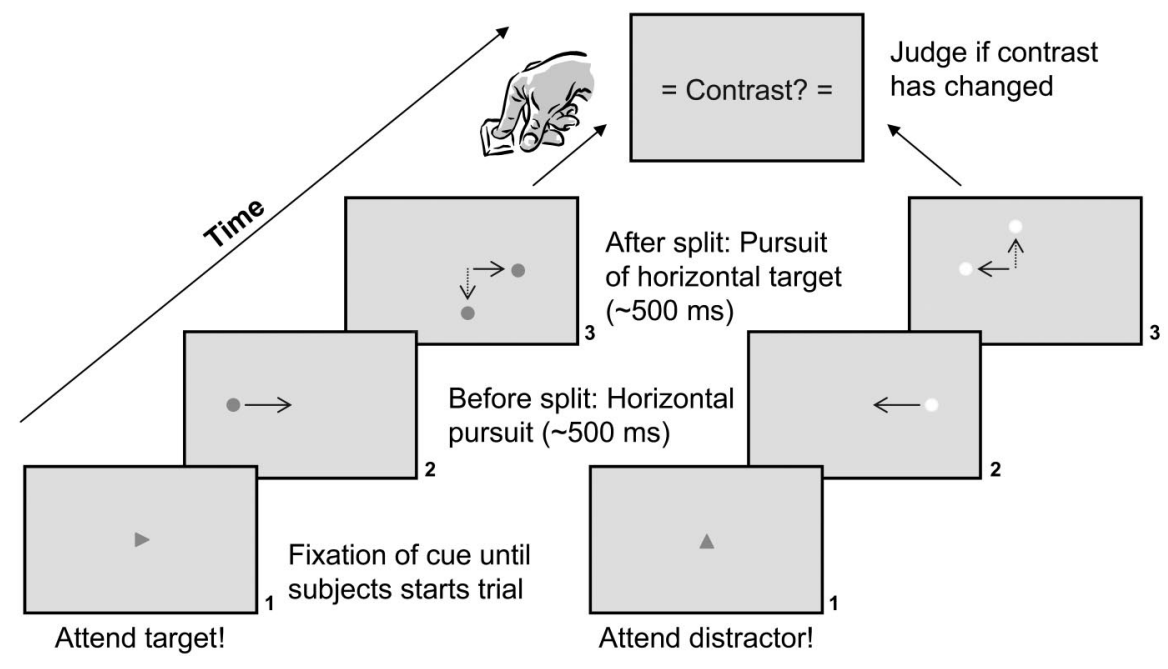

Figure 12. Sequence of events in a single trial in the cueing paradigm (Experiment 8). Each trial began with a central cue that also served as a fixation point for drift correction. The cue had equal probability of pointing to the horizontal target or to the distractor (vertically up or down) and was valid in $80 \%$ of all trials. Observers were asked to attend to the cued stimulus, while tracking the horizontal target. At the end of each trial, observers performed a forced-choice task and indicated whether target or distractor contrast had changed. Luminance (and therefore stimulus contrast to background) changed in $50 \%$ of the trials. 

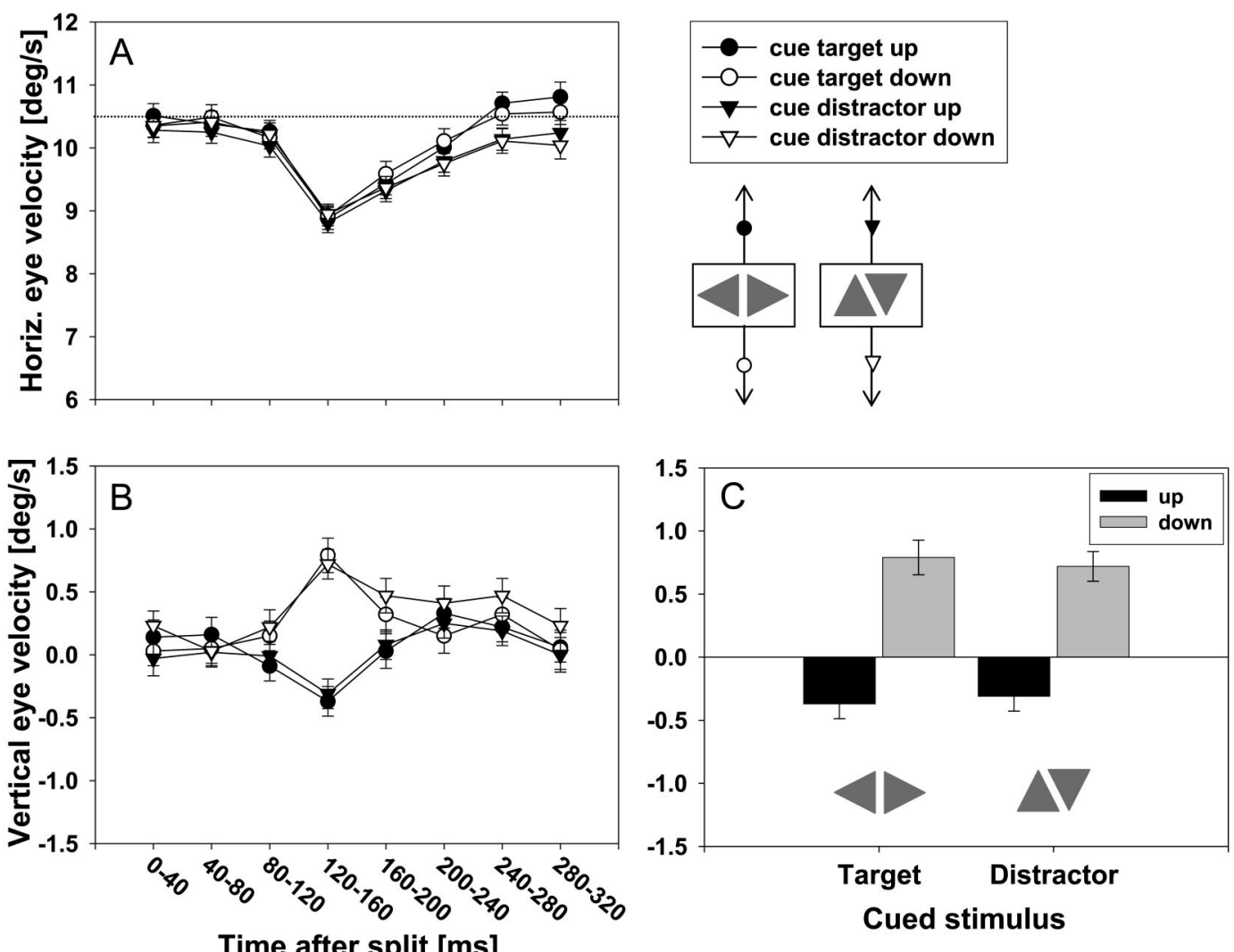

Figure 13. Results for Experiment $9(N=12)$. A: Horizontal eye velocity for time interval $0-320 \mathrm{~ms}$ for two vertical distractor directions and two cuing conditions (target or distractor cued). B: Vertical eye velocity for time interval 0-320 ms for the same conditions as in Panel A. C: Vertical eye velocity averaged across time interval $120-160 \mathrm{~ms}$, directly comparing results for a cued target to results for a cued distractor.

Condition $\times$ Distractor Direction) showed that the effect of cuing condition was not significant, $F(1,11)=0.79, p=.39$. As in the experiments before, there was a significant effect of distractor direction, $F(1,11)=50.10, p<.001$. The interaction approached significance, $F(1,11)=4.13, p=.07$, which might reflect the up-down asymmetry in the vertical deviation.

Irrespective of whether the attended location was the target or distractor, vertical eye velocity deviated away from the distractor. The influence of a moving distractor on pursuit velocity was not modulated by a voluntary covert shift of spatial attention. The premotor theory of attention alone cannot explain the effect of vertical deviation during steady-state tracking of a horizontal target in the presence of a to-be-ignored distractor.

\section{Experiment 10: Effects of Distractor Predictability}

As has already been mentioned, mislocalization of object motion onset depends on stimulus uncertainty (Müsseler \& Kerzel, 2004). In the paradigm used so far, the distractor starting position and time, as well as its motion direction, were unpredictable. In Experiment 7, we varied motion onset position along the vertical axis and therefore increased distractor uncertainty even more. Here, we test whether the effect of a vertical moving distractor on eye velocity can be diminished by reducing its spatial, temporal, and directional uncertainty.

Method. A vertical distractor appeared at the same time as a horizontally moving target and crossed the target trajectory at a random but predictable point in time. There were two distractor directions: vertically up and down. In all other aspects, the experimental paradigm was identical to the one described in Experiment 1. Six observers participated in this experiment.

Results and discussion. Figure 14 shows the effect on horizontal (Figure 14A) and vertical eye velocity (Figure 14B). We found neither a slowing in horizontal eye velocity nor a vertical deviation away from the distractor after the two stimuli crossed. There was, in fact, no vertical curvature in any of the observers tested in this experiment. A one-way repeated measures ANOVA (distractor direction) did not show a main effect of distractor direction on vertical eye velocity, $F(1,5)=2.79, p=.16$, or on horizontal eye velocity, $F(1,5)=0.65, p=.46$. Through $t$ tests, we confirmed that the vertical deviation was not significantly different from zero, for either upward, $t(5)=-2.40, p=.06$, or downward distractor motion, $t(5)=-0.08, p=.94$.

It seems that a moving vertical distractor only affected eye velocity when distractor onset position and motion direction were unpredictable. The effect vanished completely when the observer knew when and where the distractor was going to cross the target trajectory. 

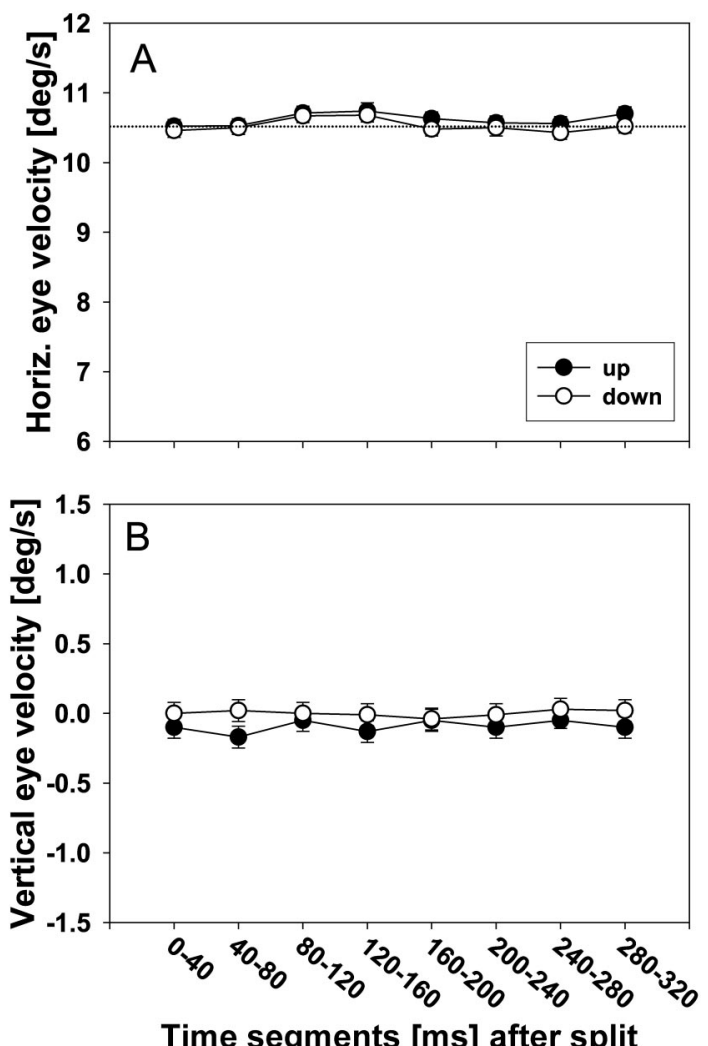

Figure 14. Results for Experiment $10(N=6)$. The distractor started at the same time as the target. A: Horizontal eye velocity for time interval 0-320 ms for two vertical distractor directions. B: Vertical eye velocity for time interval $0-320 \mathrm{~ms}$ for same conditions as in Panel A.

\section{General Discussion}

We have shown that the horizontal smooth pursuit component slowed down dramatically in response to a moving distractor stimulus that appeared during steady-state tracking. Further, we have found that the eye vertically deviated away from a moving distractor when observers were instructed to follow a horizontal target and ignore the distractor. In a series of control experiments, we have further shown that this distractor interference effect on horizontal and vertical eye velocity varied systematically with direction (Experiment 1), velocity (Experiment 4), and contrast (Experiment 5). The vertical deviation effect disappeared when two distractors were moving into vertically opposite directions (Experiment 6). The distractor interference effect was also found for vertical targets and horizontal distractors (Experiment 1) and for the initiation phase of the smooth pursuit response (Experiment 2). Furthermore, we presented some evidence that the effect does not result from a mislocalization of the distractor onset position at the time of the split (Experiment 7) and that it cannot be solely due to an illusory vertical motion component in the target (Experiment 8). The effect also does not seem to be modulated by a voluntary shift of covert spatial attention (Experiment 9). However, effects of a moving distractor on horizontal and vertical eye velocities disappeared when the observer could foresee when and where the distractor would appear (Experiment 10). When the distractor direction and its onset position were predictable, the eye followed a winner-take-all strategy. However, when distractor direction and onset were unpredictable, and the observer had prior information about target identity (Experiments 1-2, 4-9), eye movements deviated away from the distractor. Deviation away from the distractor occurred regardless of whether the distractor appeared during steady-state tracking or at pursuit initiation. In contrast, when the observer did not know in which direction the target moved (Experiment 3), eye velocity followed the vector average of target and distractor.

Taken together, these results are not consistent with the finding that eye movements generally follow the vector average of velocity or position in multiple-target situations. The results-with the exception of those of Experiment 10-also cannot be reconciled with the use of a winner-take-all strategy, which best described the steady-state phase results obtained by Recanzone and Wurtz (1999). It is interesting to note that our finding that the eye curves away from the distractor also applies to the initiation phase of the eye movement. This result shows two things: First, the eye does not naturally follow a vector averaging response during the initiation phase. Second, it cannot be the exact expectation about the target direction alone that is responsible for the deviation effect. In Experiment 2, observers knew only that the target would move horizontally (whereas the distractor always moved vertically), but they did not know whether it would move leftward or rightward. Therefore, deviation away from a second stimulus may also occur under conditions of (minimal) uncertainty.

In models of information integration for object motion perception or smooth eye movements (e.g., Adelson \& Movshon, 1982; Masson, Rybarczyk, Castet, \& Mestre, 2000; Pack \& Born, 2001) inhibition has not played a significant role so far. In most studies on target selection, especially during pursuit initiation, vector averaging has been the prevailing response. Vector averaging has also long been claimed to be the dominating strategy in the open-loop saccadic system: When observers have to decide between two adjacent stationary objects, saccades tend to land between the spatial locations of the two objects (Chou, Sommer, \& Schiller, 1999) or in the center of gravity (the global effect; Findlay, 1982).

However, our results are in line with more recent studies showing that saccades to visual targets curve away from visual distractors to which the observer had previously oriented attention (Sheliga et al., 1994). When attention was focused on a visual or acoustic stimulus in the periphery while making a horizontal or vertical saccade to a predefined target, the saccade trajectory spatially curved away from the attended location (Sheliga, Riggio, $\&$ Rizzolatti, 1995). The same results were found for visual stimuli that were ignored (Doyle \& Walker, 2001), for remembered stimulus locations (Theeuwes, Olivers, \& Chizk, 2005), and for reaching movements (Tipper et al., 1997; Tipper, Howard, \& Paul, 2001). One plausible explanation for the curvature of motion trajectories away from a distractor comes from an inhibition-based model (e.g., Tipper et al., 1997). According to the model, curvature arises during the process of target selection due to inhibition of distractor-related activity. In a first step, attention is shifted to the distractor voluntarily or involuntarily. Second, a response to the distractor is programmed but not executed because the observer is instructed to follow the target and ignore the distractor. This inhibition of the distractor response reduces the activity in neurons 
associated with the response to the distractor. If we assume, as an alternative to a vector averaging model, that the oculomotor system determines the direction and velocity of moving objects by a population response, target and distractor direction here are encoded by activity in overlapping neuronal populations. The inhibition might therefore lead to a reduced neuronal activity in a subset of neurons involved in programming a response to the target. As a result, the response to the target deviates away from the inhibited region. The effect of the pursuit eye movement curving away from a to-be-ignored distractor seemingly indicates something about visual information processing and reflects a process by which the brain ignores the distractor. However, because of its pop-out nature, the distractor cannot be ignored completely and triggers an inhibitory selection process. Therefore, it is neither a vector averaging nor a winner-take-all mechanism, but an inhibitory process, that underlies target selection in the paradigm used here. This inhibitory process comes at a cost: Horizontal eye velocity deteriorates and the eye is vertically deviated away from the distractor.

Although we ruled out the explanation that the results are due to a shift in voluntary spatial attention, we cannot rule out the effect of involuntary attention. Attention might have been captured automatically by the distractor's abrupt onset (Jonides \& Yantis, 1988) or by its motion (Franconeri \& Simons, 2003) that signals a potential behavioral priority. It is outside the scope of this article to discuss the differential effects that the allocation of voluntary versus involuntary attention might have on the eye movement response to the moving distractor (but see Prinzmetal, McCool, \& Park, 2005, for a discussion of differential effects). However, even if attention was passively captured by the distractor, it was the voluntary allocation of attention that produced the deviation effect in the Sheliga et al. (1994, 1995) studies. It therefore seems adequate to test the modulating effect of voluntary spatial attention only. In summary, we suggest that our findings, although not modulated by attention, can be explained by an inhibitory-based model that might rely on bottom-up mechanisms or other cognitive factors such as the temporal and spatial predictability of the distractor instead of voluntary spatial attention.

We are not the first to test the effect of distractors on smooth pursuit eye movements. Garbade and Deubel (2002) tested the effect of irrelevant distractors on steady-state pursuit velocity. In contrast to our experiments, target and distractor were of different form and color. This study does not reveal any effect of a vertical distractor on vertical eye velocity during horizontal smooth pursuit. The effect on the horizontal pursuit component was very small. Static distractors also do not seem to have an effect on eye velocity during the initiation phase of a smooth pursuit eye movement (Knox \& Bekkour, 2004).

The effect of a stationary, flashed stimulus on steady-state pursuit eye movements was recently tested in a study by Blohm, Missal, and Lefèvre (2005). The flashed stimulus evokes a smooth eye movement toward the flash but only in trials in which the stimulus was relevant to the task (i.e., when observers had to make a saccade to the position of the flash). This effect did not occur during fixation.

Souman, Hooge, and Wertheim $(2005,2006)$ have tested the ability to judge motion direction and localize onset position of moving objects during smooth pursuit eye movements. When observers had to indicate the direction of motion of a vertically moving stimulus while tracking a horizontal target, the motion path of the second stimulus appeared oblique into the direction opposite from the pursuit direction. In the same paradigm, the starting position of a vertically moving stimulus was mislocalized in the direction of stimulus motion. These effects are specific to self-motion and do not occur during fixation. Effects on eye position and velocity are not reported.

For the first time, our study reveals the effects of a moving distractor on the horizontal and vertical component of pursuit eye movements in response to a predefined target. Our findings agree with those regarding distractor effects on saccade trajectories. Recently, an attempt has been made to model the saccadic eye movement response to the presence of multiple stimuli (Arai \& Keller, 2005). Although this model does not take into account results showing that saccade trajectories can be curved in the direction opposite from the distractor, similar attempts should be made to model the pursuit response to multiple targets during both phases of the pursuit response, with and without previous information about target and distractor. Together with other studies, the experimental evidence provided here could be the foundation of a neurophysiologically plausible model for the pursuit response to multiple targets.

\section{References}

Adelson, E. H., \& Movshon, J. A. (1982, December 9). Phenomenal coherence of moving visual patterns. Nature, 300, 523-525.

Arai, K., \& Keller, E. L. (2005). A model of the saccade-generating system that accounts for trajectory variations produced by competing visual stimuli. Biological Cybernetics, 92, 21-37.

Blohm, G., Missal, M., \& Lefèvre, P. (2005). Direct evidence for a position input to the smooth pursuit system. Journal of Neurophysiology, 94, 712-721.

Born, R. T., Pack, C. C., \& Zhao, R. (2002). Integration of motion cues for the initiation of smooth pursuit eye movements. In J. Hyönä, D. P. Munoz, W. Heide, \& R. Radach (Eds.), The brain's eye: Neurobiological and clinical aspects of oculomotor research (Vol. 140, pp. 225237). Amsterdam: Elsevier.

Chou, I. H., Sommer, M. A., \& Schiller, P. H. (1999). Express averaging saccades in monkeys. Vision Research, 39, 4200-4216.

Desimone, R., \& Duncan, J. (1995). Neural mechanisms of selective visual attention. Annual Review of Neuroscience, 18, 193-222.

Doyle, M., \& Walker, R. (2001). Curved saccade trajectories: Voluntary and reflexive saccades curve away from irrelevant distractors. Experimental Brain Research, 139, 333-344.

Eriksen, B. A., \& Eriksen, C. W. (1974). Effects of noise letters upon the identification of a target letter in a non-search task. Perception \& Psychophysics, 16, 143-149.

Ferrera, V. P. (2000). Task-dependent modulation of the sensorimotor transformation for smooth pursuit eye movements. Journal of Neurophysiology, 84, 2725-2738.

Ferrera, V. P., \& Lisberger, S. G. (1997). Neuronal responses in visual areas MT and MST during smooth pursuit target selection. Journal of Neurophysiology, 78, 1433-1446.

Findlay, J. M. (1982). Global visual processing for saccadic eye movements. Vision Research, 22, 1033-1045.

Franconeri, S. L., \& Simons, D. J. (2003). Moving and looming stimuli capture attention. Perception \& Psychophysics, 65, 999-1010.

Fröhlich, F. W. (1923). Über die Messung der Empfindungszeit [Measuring the time of sensation]. Zeitschrift für Sinnesphysiologie, 54, 58-78.

Garbade, S., \& Deubel, H. (2002). Mechanisms of smooth pursuit eye movements after pursuit initiation [Abstract]. Journal of Vision, 2, 176. 
Groh, J. M., Born, R. T., \& Newsome, W. T. (1997). How is a sensory map read out? Effects of microstimulation in visual area MT on saccades and smooth pursuit eye movements. Journal of Neuroscience, 17, 43124330.

Heinen, S. J., \& Keller, E. L. (2004). Smooth pursuit eye movements: Recent advances. In L. M. Chalupa \& J. S. Werner (Eds.), The visual neurosciences (Vol. 2, pp. 1402-1414). Cambridge, MA: MIT Press.

Jonides, J., \& Yantis, S. (1988). Uniqueness of abrupt visual onset in capturing attention. Perception \& Psychophysics, 43, 346-354.

Kerzel, D., \& Gegenfurtner, K. R. (2004). Spatial distortions and processing latencies in the onset repulsion and Fröhlich effects. Vision Research, 44, 577-590.

Kerzel, D., \& Ziegler, N. E. (2005). Visual short-term memory during smooth pursuit eye movements. Journal of Experimental Psychology: Human Perception and Performance, 31, 354-372.

Khurana, B., \& Kowler, E. (1987). Shared attentional control of smooth eye movement and perception. Vision Research, 27, 1603-1618.

Knox, P. C., \& Bekkour, T. (2004). Spatial mapping of the remote distractor effect on smooth pursuit initiation. Experimental Brain Research, 154, 494-503.

Kowler, E. (1990). The role of visual and cognitive processes in the control of eye movement. In E. Kowler (Ed.), Eye movements and their role in visual and cognitive processes (pp. 1-70). Amsterdam: Elsevier.

Lindner, A., Schwarz, U., \& Ilg, U. J. (2001). Cancellation of self-induced retinal image motion during smooth pursuit eye movements. Vision Research, 41, 1685-1694.

Lisberger, S. G., \& Ferrera, V. P. (1997). Vector averaging for smooth pursuit eye movements initiated by two moving targets in monkeys. Journal of Neuroscience, 17, 7490-7502.

Lott, L. A., \& Post, R. B. (1993). Up-down asymmetry in vertical induced motion. Perception, 22, 527-535.

Masson, G. S., Rybarczyk, Y., Castet, E., \& Mestre, D. R. (2000). Temporal dynamics of motion integration for the initiation of tracking eye movements at ultra-short latencies. Visual Neuroscience, 17, 753-767.

Müsseler, J., \& Kerzel, D. (2004). The trial context determines adjusted localization of stimuli: Reconciling the Fröhlich and onset repulsion effects. Vision Research, 44, 2201-2206.

Pack, C. C., \& Born, R. T. (2001, February 22). Temporal dynamics of a neural solution to the aperture problem in visual area MT of macaque brain. Nature, 409, 1040-1042.

Posner, M. I. (1980). Orienting of attention. Quarterly Journal of Experimental Psychology: Human Experimental Psychology, 32(A), 3-25.

Prinzmetal, W., McCool, C., \& Park, S. (2005). Attention: Reaction time and accuracy reveal different mechanisms. Journal of Experimental Psychology: General, 134, 73-92.

Rashbass, C. (1961). The relationship between saccadic and smooth tracking eye movements. Journal of Physiology, 159, 326-338.

Recanzone, G. H., \& Wurtz, R. H. (1999). Shift in smooth pursuit initiation and MT and MST neuronal activity under different stimulus conditions. Journal of Neurophysiology, 82, 1710-1727.
Recanzone, G. H., \& Wurtz, R. H. (2000). Effects of attention on MT and MST neuronal activity during pursuit initiation. Journal of Neurophysiology, 83, 777-790.

Rizzolatti, G., Riggio, L., Dascola, I., \& Umiltà, C. (1987). Reorienting attention across the horizontal and vertical meridians: Evidence in favor of a premotor theory of attention. Neuropsychologia, 25, 31-40.

Schlag, J., \& Schlag-Rey, M. (2002). Through the eye, slowly: Delays and localization errors in the visual system. Nature Reviews Neuroscience, 3, 191-215.

Sheliga, B. M., Riggio, L., \& Rizzolatti, G. (1994). Orienting of attention and eye movements. Experimental Brain Research, 98, 507-522.

Sheliga, B. M., Riggio, L., \& Rizzolatti, G. (1995). Spatial attention and eye movements. Experimental Brain Research, 105, 261-275.

Souman, J. L., Hooge, I. T., \& Wertheim, A. H. (2005). Vertical object motion during horizontal ocular pursuit: Compensation for eye movements increases with presentation duration. Vision Research, 45, 845853 .

Souman, J. L., Hooge, I. T., \& Wertheim, A. H. (2006). Localization and motion perception during smooth pursuit eye movements. Experimental Brain Research, 4, 448-458.

Spering, M., Kerzel, D., Braun, D. I., Hawken, M. J., \& Gegenfurtner, K. R. (2005). Effects of contrast on smooth pursuit eye movements. Journal of Vision, 5, 455-465.

SPSS, Incorporated (2001). SPSS 11.0 for Windows [Computer software]. Chicago, IL: Author.

Stroop, J. R. (1935). Studies of interference in serial verbal reactions. Journal of Experimental Psychology, 18, 643-662.

Theeuwes, J., Olivers, C. N., \& Chizk, C. L. (2005). Remembering a location makes the eyes curve away. Psychological Science, 16, 196199.

Thompson, P. (1982). Perceived rate of movement depends on contrast. Vision Research, 22, 377-380.

Thornton, I. M. (2002). The onset repulsion effect. Spatial Vision, 15 219-243.

Tipper, S. P., Howard, L. A., \& Jackson, S. R. (1997). Selective reaching to grasp: Evidence for distractor interference effects. Visual Cognition, 4, 1-38.

Tipper, S. P., Howard, L. A., \& Paul, M. A. (2001). Reaching affects saccade trajectories. Experimental Brain Research, 136, 241-249.

Wyatt, H. J., Pola, J., \& Lustgarten, M. (1989). The oculomotor "twitch": A transient response to target motion. Experimental Brain Research, 76, $581-592$.

Zhou, W., \& King, W. M. (2002). Attentional sensitivity and asymmetries of vertical saccade generation in monkey. Vision Research, 42, 771-779.

Received October 7, 2005

Revision received January 26, 2006

Accepted March 12, 2006 